\title{
ATAC-Seq and RNA-Seq combined analysis elucidates the impact of BORIS/CTCFL on melanoma progression, mediated by chromatin accessibility and gene expression alterations
}

\author{
Roy Moscona ${ }^{1}$, Sanne Marlijn Janssen ${ }^{2,3}$, Mounib Elchebly ${ }^{2}$, Andreas Ioannis Papadakis ${ }^{2}$, Alan \\ Spatz $^{2,3,4,5}$ and Eitan Rubin ${ }^{1}$ \\ ${ }^{1}$ The Shraga Segal Department of Microbiology, Immunology and Genetics, Ben-Gurion University of the Negev, Beer Sheva, Israel. ${ }^{2}$ Lady Davis Institute for \\ Medical Research, Montréal, QC, Canada. ${ }^{3}$ Department of Pathology, McGill University, Montréal, QC, Canada. ${ }^{4}$ Division of Pathology, Department of \\ Laboratory medicine, McGill University Health Center, Montreal, QC, Canada. ${ }^{5}$ Department of Oncology, McGill University, Montréal, QC, Canada
}

\begin{abstract}
Melanoma accounts for most deaths form skin cancer, since it metastasizes early, and it usually diagnosed at an advance stage. Brother of Regulator of Imprinted Sites (BORIS), also known as CCCTC binding factor-Like (CTCFL), is a transcriptional modulator that becomes aberrantly expressed in melanoma. Yet, the role of BORIS in melanoma remains elusive, in our latest study we sought to uncover its effect on the biological processes and pathways in melanoma cells lines, by using a doxycycline inducible system expressing ectopic BORIS, we investigated its effect on chromatin accessibility at genes promoters and transcriptomics changes, utilizing Assay for Transposase-Accessible Chromatin using sequencing (ATAC-Seq) and RNA-Seq respectively. A differential analysis revealed BORIS target genes and transcription factors (TFs) it governs, in its role as a tumor driver factor. It targets include an over representation of oncogenes, specially upregulated tumor progressors associated with an invasive phenotype such as PDL-1, CTLA4 and GDF6, a known metastatic driver gene. In addition, activators TFs include TEAD4, STAT6 and SP1, while repressed TFs are known tumor suppressors, such as P53,P73 and SOX10. Together, these findings situate BORIS as a potential factor that can lead to an invasiveness phenotype, which drive melanoma progression towards metastatic dissemination.
\end{abstract}




\section{INTRODUCTION}

Primary cutaneous melanoma accounts for most of the deaths from skin cancer in the united states, due to its ability to quickly disseminate and form distant metastases (Siegel, Miller, \& Jemal, 2020). In the progression of primary tumor towards an aggressive metastatic stage, cells undergo a reversible transition from proliferative to invasive states. This change is known as "phenotype switching", and it resembles epithelial-mesenchymal transition (EMT) (Hoek, Eichhoff, et al., 2008; Hoek \& Goding, 2010). Studies of melanoma identified specific genes and transcription factors (TFs) involvement in different stages of the disease (Hoek et al., 2006; Jeffs et al., 2009; Tirosh et al., 2016; Widmer et al., 2012). The 'proliferative' state of melanoma cells was linked to high expression levels of TFs such as the melanocyte differentiation gene MITF (Hoek, Schlegel, et al., 2008), SOX10 (Shakhova et al., 2012) and PAX3 (He, Stevens, Braithwaite, \& Eccles, 2005). On the contrary, melanocytes of the 'invasive' signature express low levels of 'proliferative' signature TFs and higher levels of different TFs such as TEAD (Verfaillie et al., 2015), BRN2 (Pinner et al., 2009), Activator Protein-1 (AP-1) family (Jochum, Passegué, \& Wagner, 2001), NFATC2 (Perotti et al., 2019) and NFIB (Fane et al., 2017). Epigenetic studies of melanoma-genesis revealed chromatin landscape changes in different phenotypic states of the disease (Dahl \& Guldberg, 2007; Fiziev et al., 2017; Verfaillie et al., 2015). The genetic and epigenetic alterations are driven by transcriptional regulators. Exploring their exact mechanism of action in melanoma is pivotal for our understanding of the development of the disease. An important chromatin modifier in malignancies is the Brother of Regulator of Imprinted Sites (BORIS) also known as CTCFL (CCCTC binding factor-Like). It influences the chromatin landscape and transcription, both directly and indirectly. BORIS' role in various cancer types has been studied. Yet, the knowledge of BORIS function in melanoma is incomplete, it has been suggested to play a key role in tumor progression, with high expression levels of BORIS in 59\% of melanoma cell lines, $16 \%$ of primary melanoma and $34 \%$ of melanoma metastasis. Despite these evidence, BORIS exact mechanism of action in melanoma development and progression is yet to be deciphered, as studies of it in the context of melanoma are scarce.

BORIS is a DNA-binding protein with high similarity to its sibling gene CTCF (Loukinov et al., 2002), the multifunctional 'master weaver' of the genome transcriptional factor, with has a great influence on chromatin organization (Phillips \& Corces, 2009). Under normal conditions, BORIS functional expression was found to be strictly restricted to the testis, whilst it becomes aberrantly expressed in different types of cancers (Loukinov et al., 2002), being the most frequently switched on cancer testis antigen (CTA) gene (Wang et al., 2016). The delicate balance between the sibling genes is shifted towards BORIS in malignant cells (Klenova, Morse, Ohlsson, \& Lobanenkov, 2002). Unlike CTCF's tumor suppressor properties (Kemp et al., 2014; Rasko et al., 2001), BORIS role as an oncogene, has been shown in different malignancies such as breast, prostate and colorectal cancer (Cheema et al., 2014; D’Arcy et al., 2008; Dougherty et al., 2008; Gaykalova et al., 2012; Q. Liu et 
al., 2017; Renaud et al., 2011; Smith et al., 2009; Yanmei Zhang et al., 2017). In ovarian cancer BORIS been shown to increase invasiveness(Hillman et al., 2019). We have shown in a previous study that BORIS ectopic expression in melanoma cells reduced proliferation and increased invasiveness properties, demonstrated by a transcriptional switch accompanied by a phenotypic switch (Janssen et al., 2020).

Here, we explore the impact of BORIS ectopic expression on melanoma cells chromatin accessibility and transcriptional landscape. We utilized Assay for Transposase-Accessible Chromatin using sequencing (ATAC-Seq) (Buenrostro, Giresi, Zaba, Chang, \& Greenleaf, 2013) to understand genome wide chromatin accessibility changes induced by BORIS. Based on chromatin analysis of ATAC-Seq, combined with gene expression data of RNA-Seq, employing a doxycycline (DOX)inducible expression system in melanoma cells, we revealed BORIS target genes and TFs, which it regulates, thus contributing to tumor invasiveness and progression, which eventually lead to metastasis dissemination in melanoma. We found BORIS ectopic expression differently upregulates the promoter region and mRNA transcripts of key regulator genes, among them; PDL-1, GDF6, CTLA4, CDX2. At the same time, TFs that govern the invasive phenotype such as TEAD4, STAT6, NFYB and AP-1 and NFAT families are also upregulated by BORIS, while differentially downregulated TFs include the proliferative marker SOX10 and well-known tumor suppressors P53 and P73. In addition, enrichment analyses of genes with a differential accessible promoter region, showed high enrichment in invasiveness related biological processes. Overall, our data indicate a unique BORIS genetic signature in melanoma cells, defined by the genes and TFs it govern in its role as a regulator of chromatin accessibility and gene transcription, in a manner which drives melanoma tumor progression towards metastasis.

\section{METHONS}

\subsection{Cell lines}

Early passage MM057 melanoma cells were a kind gift from Dr. G. Ghanem (Institut Jules Bordet, Brussels, Belgium) and were cultured in Ham'sF10 medium supplemented with $8 \%$ heat-inactivated fetal bovine serum, $1 \%$ penicillin-streptomycin and $1 \%$ GlutaPlus. Doxycycline inducible cells were selected by the addition of $150 \mu \mathrm{g} / \mathrm{ml} \mathrm{G} 418$ (Neomycin) for a minimum of 2 weeks or until noninfected control cells were killed. Upon selection, MM057 cells were maintained in the presence of 50 $\mu \mathrm{g} / \mathrm{ml} \mathrm{G} 418$. Doxycycline (dox; Clontech) was added to the medium for the indicated dose and time and refreshed every $48 \mathrm{~h}$. Bimonthly tests for mycoplasma demonstrated the absence of contamination. All cultures were maintained at $37^{\circ} \mathrm{C}$ in a $5 \% \mathrm{CO} 2$ humidified atmosphere.

\subsection{Generation of expression vectors}


Full-length human BORIS cDNA was amplified by PCR on RNA extracted from HEK293T cells using iProof (Bio-Rad) with primers containing BamHI and MluI restriction sites (forward 5'CAGCGGATCCACTGAGATCTCTGTCCTTTCTGAG-3' and reverse 5'-GCGGACGCGTCT CACTTATCCATCGTGTTGAGGAGCATTTCACAGG-3'). The PCR product was digested with BamHI and MluI (Fermentas FastDigest) and inserted into pDONR221 (Invitrogen cat \# 12536017) by a BP reaction (GatewayTM BP ClonaseTM II Enzymemix, Invitrogen) to generate pENTR-BORIS with six C-terminal His-tags followed by a stop codon. To generate pI20-BORIS-6xH the entry clone was recombined into the lentiviral destination vector pI20 (empty vector (EV)-6xH, a kind gift from Stephen Elledge(Meerbrey et al., 2011), Addgene plasmid \#44012) using Gateway LR ClonaseTM II (Fisher).

\subsection{Transfection, lentivirus production, and infection}

For virus production, pI20-BORIS-6xH or pI20-EV-6xH lentiviral expression vector (10 $\mu \mathrm{g}$ DNA) was transfected into HEK293T cells along with the viral packaging plasmids pSPAX2 (7.5 $\mu$ g DNA) and pMD2.G (5 $\mu \mathrm{g}$ DNA) using polyethyleneimine (PEI; Polysciences, DNA:PEI ratio of 1:2.5) and Opti-MEM transfection medium (Invitrogen). The medium was replaced by fresh medium $3 \mathrm{~h}$ after transfection. At $48 \mathrm{~h}$ post transfection, virus-containing supernatant was harvested, centrifuged, passed through a $0.45 \mu \mathrm{m}$ syringe filter, and either used directly for infection or stored at $-80^{\circ} \mathrm{C}$. Cells were infected twice by incubation with a mixture of viral supernatant, medium (1:1 ratio) and polybrene (8 $\mu \mathrm{g} / \mathrm{ml}$, Sigma-Aldrich) for $12 \mathrm{~h}$. The viral mixture was replaced by fresh medium for $24 \mathrm{~h}$ before selection. Expression of the constructs was verified by a combination of immunoblot and quantitative PCR.

\subsection{RNA extraction, reverse transcription, and quantitative PCR}

Total RNA was extracted using the Nucleospin RNA kit (Macherey Nagel) according to manufacturer's instructions. DNase treatment excluded genomic DNA. RNA quantity was determined by NanoDrop. Total RNA (400 ng) was reverse transcribed using the qScript cDNA synthesis kit (Quanta) according to manufacturer's specifications. qPCR was performed with PerfeCTa SYBRGreen FastMix (Quanta) on a CFX96 TouchTM (Bio-Rad). Each $10 \mu 1$ reaction contained $4 \mu \mathrm{l}$ cDNA (1/5 diluted), $5 \mu$ PCR mix and 400nM forward and reverse primer. The thermal cycling program used was as follows: $30 \mathrm{sec}$ at $95{ }^{\circ} \mathrm{C}$ followed by 40 cycles of $5 \mathrm{sec}$ at $95{ }^{\circ} \mathrm{C}$ and $20 \mathrm{sec}$ at 60 ${ }^{\circ} \mathrm{C}$, followed by $10 \mathrm{sec}$ at $95{ }^{\circ} \mathrm{C}$ and a melt curve with incremental steps of $0.5{ }^{\circ} \mathrm{C}$ for $5 \mathrm{sec}$ starting at $65^{\circ} \mathrm{C}$ and ending at $95{ }^{\circ} \mathrm{C}$. Primers were designed to be exon spanning when possible. For each primer pair, the melting temperature, GC content and the secondary structure of the PCR product were assessed using tools available from the University of California Santa Cruz Genome Browser (https://genome.ucsc.edu/cgi-bin/hgPcr) and Integrated DNA Technologies (https://www.idtdna.com). Melting curve analyses were carried out to ensure product specificity. Primers were purchased from 
Integrated DNA Technologies and sequences are provided in Supp 9-10 . Importantly, BORIS primers span exon 9 and 10, and were designed to amplify isoform family's sf1 and sf5 (Pugacheva et al., 2010). Samples were run in technical triplicate and data were normalized to both HPRT1 and TBP. All data were analyzed using the CFX ManagerTM Software (Bio-Rad).

\subsection{Immunoblot}

Cells were grown to $70 \%$ confluency and harvested in ice cold PBS by scraping. The collected cell pellets were lysed on ice with lysis buffer (50 mM HEPES-KOH pH7.4, $150 \mathrm{mM} \mathrm{NaCl,} 1 \%$ triton X100, $0.2 \%$ sodium deoxycholate, $5 \mathrm{mM}$ EGTA, $10 \%$ glycerol) supplemented with 1x protease inhibitor cocktail (Promega) and phosphatase inhibitor (Roche) and sonicated three times for $15 \mathrm{sec}$ at $25 \%$ power. Lysates were cleared by centrifugation and protein concentration was determined using the Pierce BCA protein assay kit (Fisher) according to manufacturer's instructions. Lysates were denatured in SDS sample buffer for $5 \mathrm{~min}$ at $95{ }^{\circ} \mathrm{C}$. For direct lysis, $2 \times 105$ cells were lysed in $1 \mathrm{x}$ SDS sample buffer and denatured for $10 \mathrm{~min}$ at $95^{\circ} \mathrm{C}$. Protein extracts were separated on SDSpolyacrylamide gel and transferred onto polyvinylidene difluoride membranes (Bio-Rad). Membranes were blocked with 3\% non-fat milk (Bio-Rad) or 3\% bovine serum albumin (Bioshop) in PBS containing $0.05 \%$ Tween 20 (TBST) and probed with the appropriate primary antibody overnight at 4 ${ }^{\circ} \mathrm{C}$. Blots were washed with TBST and probed with the corresponding horseradish peroxidaseconjugated secondary antibody. Proteins were detected using enhanced chemiluminescence reagent (Bio-Rad), images were captured with a ChemiDocTM Imaging System (Bio-Rad) and analyzed with Image LabTM Software (Bio-Rad Laboratories, Version 5.0 build 18). The following antibodies were used: rabbit anti-human BORIS (Rockland, 600-401-907) at a 1:1000 dilution and rat anti-human tubulin (Abcam, ab6160) at 1:7000. Secondary antibodies used were goat anti-rabbit (Jackson, 115035-144) and anti-rat IgG (Cell Signaling, 7077) coupled to horseradish peroxidase.

\subsection{Assay for transposase accessible chromatin followed by sequencing (ATAC-seq)}

For ATAC-seq library preparation cells were detached from the culture dish with citric saline ( $270 \mathrm{mM} \mathrm{KCl}, 30 \mathrm{mM}$ sodium citrate), passed through a 40um filter and counted using a hemocytometer to collect 50.000 cells for all four experimental conditions. To isolate nuclei, cells were first lysed for $20 \mathrm{~min}$ on ice in lysis buffer 1 ( $0.1 \%$ Sodium citrate tribasic dehydrate (Sigma, S1804) and 0.1\% Triton X-100 (Bioshop, TRX777.100)) followed by $20 \mathrm{~min}$ lysis on ice in lysis buffer 2 (10mM Tris-HCl pH7.4, 10mM NaCl, 3mM MgCl, 0.1\% Igepal CA630 (Bioshop, NON999.500) and 0.1\% Tween-20 (Bioshop, TWN510.500). The nuclei were pelleted by centrifugation for $10 \mathrm{~min}$ at $600 \mathrm{~g}\left(4^{\circ} \mathrm{C}\right)$ and supernatant was carefully removed to reduce mitochondrial DNA (mtDNA) content. For the transposase reaction, the nuclei pellet was resuspended in $25 \mu \mathrm{l}$ transposase reaction mix containing of $4 \mu \mathrm{l} \operatorname{Tn} 5$ transposase in $1 \mathrm{x}$ transposase reaction buffer (Nextera DNA library kit, Illumina, FC-121-1030) and 0.1\% Tween-20 (Bioshop, 
TWN510.500), and incubated for $30 \mathrm{~min}$ at $37^{\circ} \mathrm{C}$ with gentle shaking (400rpm). Immediately following transposition, DNA was purified using the MinElute PCR purification Kit (Qiagen, 28004), according to manufacturer's instructions. Library fragments were amplified in two steps. First, 5 cycles of PCR were performed using NEBNext® High-Fidelity PCR master mix (New England BioLabs, M0541) with 1.25 $\mu \mathrm{M}$ barcoded Nextera PCR primers (Supp 10.). in a final volume of $50 \mu \mathrm{l}$ on a BioRad C1000 Thermal Cycler. The cycling program used was as follows: $5 \mathrm{~min}$ at $72^{\circ} \mathrm{C}, 30 \mathrm{sec}$ at $98^{\circ} \mathrm{C}, 5$ times cycling for $10 \mathrm{sec}$ at $98^{\circ} \mathrm{C}, 30 \mathrm{sec}$ at $63^{\circ} \mathrm{C}$ and $1 \mathrm{~min}$ at $72^{\circ} \mathrm{C}$. To reduce $\mathrm{GC}$ and size bias during library amplification, $5 \mu \mathrm{l}$ from the PCR mix was used for a $15 \mu \mathrm{l}$ qCR side-reaction containing the same reagents with the addition of SYBRgreen (9X, Life Technologies, S7563) to determine the number of additional PCR cycles and stop amplification prior to saturation. The CFX96 TouchTM (Bio-Rad, 1855196) qPCR machine was used with the following cycling conditions: $30 \mathrm{sec}$ at $98^{\circ} \mathrm{C}, 20$ times cycling for $10 \mathrm{sec}$ at $98^{\circ} \mathrm{C}, 30 \mathrm{sec}$ at $63^{\circ} \mathrm{C}$ and $1 \mathrm{~min}$ at $72^{\circ} \mathrm{C}$ followed by melt curve analysis. Using the CFX ManagerTM Software (Bio-Rad), the fluorescent intensity was plotted versus cycle number and the additional number of cycles was established as the cycle number corresponding to $1 / 4$ th of the maximum fluorescent intensity. Next, the initial PCR reaction mix was amplified for 3 additional PCR cycles. Library preparation for each experimental condition was performed in triplicate and samples were combined post PCR amplification for quality controls and sequencing. One third of the library was cleaned up using KAPA Pure Beads (1.8X; Roche, 7983298001) and analyzed on a $2.5 \%$ agarose (Bio-Rad, 1613101) gel to verify nucleosomal patterning. The remainder of the library was size selected using KAPA Pure Beads (0.55X-1.8X; Roche, 7983298001) to enrich for 150-750 bp fragments. Part of the DNA from the size selected library was diluted 1 in 5 and used for qPCR to confirm enrichment for open chromatin and estimate mtDNA content. The qPCR reaction contained 2.5 $\mu$ l DNA template, 5ul PerfeCTa SYBR Green FastMix (Quanta, 95072-012) and 300nM forward and reverse primer. The thermal cycling program used was as follows: 3 minutes at $95^{\circ} \mathrm{C}, 40$ times cycling for 15 seconds at $95^{\circ} \mathrm{C}$ and 30 seconds at $60^{\circ} \mathrm{C}$ followed by melt curve analysis. To assess enrichment of open chromatin primers were designed in genomic regions that are often marked by open chromatin (GAPDH and KAT6B) or closed chromatin (SLC22A3 and RHO). A minimum difference in quantitation cycle $(\mathrm{Cq})$ value of 3 , representing an 8-fold enrichment, was considered as enrichment of open chromatin in the libraries. In addition, we performed qPCR to approximate the level of mtDNA in our libraries (established from purified and washed nuclei) as well as libraries prepared from whole cell lysate using 2 sets of primers that are referred to as mtDNA and ND1mtDNA. The sequences of these primers as well as the primers directed at mtDNA are listed in Supp 10.). The remaining DNA of the libraries was submitted to the McGill University and Genome Québec Innovation Centre and fragment distribution was assessed by TapeStation (Agilent), libraries were quantified using Quant-iTTM PicoGreen ${ }^{\circledR}$ dsDNA Reagent ${ }^{\circledR}$ (ThermoFisher Scientific), pooled and sequenced paired-end (100bp read length) on one lane of an Illumina HiSeq4000. 


\subsection{A bioinformatics pipeline for extracting high-coverage genomic regions from ATAC-Seq data}

To gain insight into the impact of BORIS on chromatin arrangement in melanoma, we utilized ATAC-Seq with two biological replicates over the four experimental conditions: BORpos, BORneg, EVpos, EVneg. ATAC-Seq data, as raw FastQ reads files, were first examined for quality assurance with FastQC(Andrews, Krueger, Seconds-Pichon, Biggins, \& Wingett, 2010). The reports indicated a couple of problematic sequencing results in the following fields: Per tile sequence quality and Adapter Content contamination. Each abnormal finding was removed before any further analysis. First, tiles that contained low-quality reads were removed using the filterbytile.sh script from BBMap tool (Bushnell B., 2014), default parameters were used. Second, an adapter contamination of the Nextera Transposase Sequence was removed, using the trim_adapters script by the attack toolkit (Parker, 2015) for ATAC-seq data, default parameters were used. Besides, the first 17 base-pairs (bp) of all reads were trimmed with cutadapt (Martin, 2011) due to poor per base sequence content. The trimmed reads had a sequence length of $83 \mathrm{bp}$ and an average of 164,561,210 (Range; 164,424,288-

$187,302,698)$ paired end (PE) total sequences, for all four samples conditions.

The next step was aligning the trimmed reads to the human reference genome GRCh38.p12 using BWA-MEM (Li, 2013), with the following parameters; bwa mem -M -a -t $30 \mathrm{hg} 38$.fa. read1.fastq read2.fastq. The aligned reads were saved as Binary Alignment Map (BAM) and further indexed and name sorted with SAMtools(Li et al., 2009). After the reads were aligned and sorted, duplicate alignments were marked and removed with Picard Tools (Wysoker, Tibbetts, \& Fennell, 2009) MarkDuplicates tool, with the following command; picard.jar MarkDuplicates I=file_name.bam O=file_name.no_duplicates.bam M=file_name.txt REMOVE_DUPLICATES=true VALIDATION_STRINGENCY=LENIENT. The average read pair duplication rate (including PCR and optical duplicates), as determined by MarkDuplicates was 31.88\% (Range; 30.2\%-35.1\%). After duplication removal, an additional filtering step was implemented with SAMtools with the following command; samtools view -b -h -f 2 -f 3 -F 4 -F 8 -F 256 -F 1024 -F 2048 -q 20 -e chrM -e chrY -e 'VN:' file_name.bam.

At the end of this sifting step, we excluded reads mapped to Mitochondrial DNA and Chromosome Y, in addition to reads that were not mapped/paired properly or mapped to multiple places in the genome, lastly reads with Q-score<20 were removed as well. The final autosomal properly aligned sequences (described in the results) for downstream analyses were saved in BAM format. The correlation between each of the two replicates of each one of the four conditions was calculated with deepTools2(Ramírez et al., 2016) with the following two commands ; (1) multiBamSummary bins -bamfiles BAM_file_1.bam BAM_file_2.bam -o Condition.npz -p "max" --ignoreDuplicates -minMappingQuality 20 ; (2) plotCorrelation --corData Condition.npz --corMethod spearman -whatToPlot scatterplot --labels Condition_1 Condition_2 -o Condition_name.pdf -- 
outFileCorMatrix Condition_file_name.

The sifted BAM served as input for MACS2(Feng, Liu, Qin, Zhang, \& Liu, 2012; Yong Zhang et al., 2008). The aim of this analysis was seeking for regions with an abundance of transposition events, which indicate OCRs. The MACS2 call-peak function was used with the following command; macs2 callpeak -g hs -q 0.05 -f BAMPE -B. The results of MACS2 were saved as BedGraph files, in addition to excel files that contain peaks location, length, enrichment and statistic-index values. From the BedGraph files, we generated UCSC Genome Browser compatible BigWig files with the following commands; (1) env LC_COLLATE=C sort -k1,1 -k2,2n file_name.bdg > file_name.sorted.bdg (2) bedGraphToBigWig file._name.sorted.bdg hg38.chrom.sizes.txt file_name.bw. Blacklisted Regions were removed as defined by the ENCODE file ENCFF419RSJ.bed.

\subsection{ATAC-seq data downstream analyses with $\mathbf{R}$ and Bioconductor}

The sifted reads (as BAM files and the MACS2 output NarrowPeak files) served as input for the analyses in R Programing language(R Core Team, 2014) with Bioconductor(Huber et al., 2015) packages. First, we checked the distribution of the final reads across all chromosomes. In general, as chromosome size increases so does the number of reads map to it, with chr1 representing the highest number of reads mapped. ATAC-seq should represent a mix of fragment lengths corresponding to nucleosome-free (NFR) positions (<100bp), mono-nucleosome (180-247bp) and poly-nucleosome fractions (>315bp) as defined by the Greenleaf lab(Buenrostro et al., 2013; Schep et al., 2015). By plotting the insert size (Supp 8.), we verified our reads did contain the expected typical ATAC-Seq pattern distribution. All samples were of the desired insert size distribution with the NFR region has the most reads, with a decreasing number of reads as insert sizes get larger. First, ATAC-Seq fragment reads of length >100bp were discarded, which may not represent Nucleosome Free Regions (NFRs). Here, we focused on the remaining fragments reads NFRs fragments (<100bp length), these fragments were plotted in a Principal component analysis (PCA) (Supp 4.). The PCA plot represents all the 8 ATAC-Seq replicates samples, as expected, the BORpos samples cluster together, apart from the rest of the samples. Variance in PC1 explains majority (85\%) of difference between samples conditions, while on PC2 samples cluster by batch effect. The PCA was plotted with DESeq2(Love, Huber, \& Anders, 2014) in R software. Non-redundant peaks were defined only as peaks which are present in both replicates (two samples) on the same tested condition. Only peaks who meet criteria were continued to assessment of changes in ATAC-seq signal of NFRs. The Sifted ATAC-Seq reads, as MACS2 NarrowPeak output peaks, were annotated to their nearest corresponding gene. In addition, a genomic region was assigned to each peak with (Figure 1b). All annotation were done using ChIPseeker (Yu, Wang, \& He, 2015) in R. Differential Accessible Regions (DARs) analysis was performed, to evaluate changes in ATAC-seq signal between BORIS samples to Empty Vector controls. Peaks which were found to be differentially accessible between the two tested conditions were defined as DARs. Thus, enabling us to identify differences in ATAC-seq signal between the two 
tested conditions. Ambiguous 196 DARs, which showed significant increased and decreased accessibility of the promoter region of a gene, were discarded from any further analyses.

\subsection{Functional analysis of statically significant upregulated promoters (0-3kb) DARs regions with Genomic Regions Enrichment of Annotations Tool (GREAT)}

Upregulated DARs that annotated to promoters $(0-3 \mathrm{~kb}$ from TSS as defined in $\mathrm{hg} 38$ reference genome) regions, included 3,573 genomic regions with positive $\log _{2} \mathrm{FC}>0$ and $\mathrm{FDR}<0.05$ when comparing BORpos to EVpos samples. The selected regions as BED file served as input for rGREAT softweare (version 4) (McLean et al., 2010; Zuguang Gu, 2020) to find enriched GO (Gene Ontology) terms for OCRs mapped to promoters (0-3kb) and defined as DARs. GO terms for BP (Biological Process), MF (Molecular Function) and CC (Cellular Component) were used. For the genes coverage of each GO term and FDR the hypergeometric test results were taken

\subsection{Gene Set Enrichment Analysis with (GSEA) of all genes with their promoter (0-}

\section{$3 \mathrm{~kb})$ region defined as DAR}

A ranked gene list was established based ATAC-Seq reads of OCRs-NFRs mapped to promoters (0$3 \mathrm{~kb}$ ) and defined as DARs. The sum of the total genes was 16,160 The gene list was ranked according to $\log _{2} \mathrm{FC}$ of each promoter (0-3kb) DAR region, as captured by the comparison between EVpos to BORpos samples. The generated gene list served as input to GSEA-pre-ranked tool (with no static cut-off as recommended), with $10^{4}$ permutations.

\subsection{Transcription Factors differential activity analysis with DiffTF}

In order to define differential TFs activity based on the chromatin accessibility data (ATAC-seq) and its integration with gene expression data (RNA-seq) for classification of TFs as genetic activators or repressors, we used a computational framework called diffTF (Berest et al., 2019). This tool searches for overlaps between OCRs-open chromatin regions from ATAC-seq and predicted TF binding sites defined by the genome-wide Position Weight Matrix (PWM) scanner, PWMscan (Ambrosini, Groux, \& Bucher, 2018). For the PWM database source, we used HOCOMOCOv11 database (Kulakovskiy et al., 2018). This database is dependent on collections of TF binding motifs of available $769 \mathrm{TFs}$, and it is not applicable to TFs with poorly defined or unknown DNA-binding motifs. DiffTF default settings were used, the signal around TF binding sites was extended by $100 \mathrm{bp}$ in each direction and calculated a fold change of chromatin accessibility, based on ATAC-Seq reads, between the two tested conditions (BORIS Vector VS Empty Vector). Bootstrapping of $10^{3}$ was used as recommended to account for differences in GC content at the different binding sites. The index for $\Delta \mathrm{TF}$ activity is the Weighted Mean Difference, with a positive value meaning the TF is activated in the condition tested upon, here, BORIS ectopic expression. Negative values mean the TF is less active in the tested 
condition (BORpos). Here, we investigated only TFs with positive values of Weighted Mean Difference since they indicate a TF to be activated by BORIS ectopic expression.

\section{RESULTS}

\subsection{Identification of differentially accessible regions (DARs) between melanoma cells expressing ectopic BORIS vector and an empty vector}

To study the effect of ectopic BORIS expression on chromatin accessibility we used a melanoma cell line (MM057), with doxycycline (dox)-inducible vector that either carried BORIS (BOR) or an Empty vector (EV), as established previously (Janssen et al., 2020). This cell line expresses low levels of BORIS mRNA, and undetectable BORIS protein, making it a suitable model system for this study. Expression of BORIS or EV was induced by culturing the cells for five days in the presence of 50ng/ml dox, as determined previously (Janssen et al., 2020). The experimental design was divided by the presence of dox as follows; positive (pos) or negative (neg) to dox presence. The four experimental sets were named: BORpos, BORneg, EVpos, EVneg. Next, ATAC-Seq (Buenrostro et al., 2013) was performed with two biological replicates for each tested condition. For all samples, we obtained $81.77 \% \pm 8.3$ (mean \pm standard deviation(sd)) mappability and $48,758,922 \pm 22.9 * 10^{6}$ final properly paired reads for downstream analyses. A high correlation was observed for all sample replicates $\left(\mathrm{R}^{2}=0.98 \pm 4 * 10^{-4}\right)$ based on the number of reads mapping to the same location in the ATACSeq data (Supp 3.), assuring that ATAC-Seq results are reproducible.

To identify Differentially Accessible Regions (DARs) between BORpos and EVpos, we first determined ATAC-Seq peaks of Open Chromatin Regions (OCRs) from nucleosome free regions (NFRs) for each experimental condition. Next, open chromatin regions peaks that showed differential accessibility between BORpos vs EVpos were obtained, revealing 158,534 peaks. Correction for multiple testing using the Benjamini-Hochberg False Discovery Rate (FDR) (Benjamini \& Hochberg, 1995 ) with a cut-off FDR $<0.05$, classified 22,605 of the peaks as significant DARs, meaning peaks that were statically significant between the two tested conditions (Figure1a). Annotation of the significant DARs to genomic regions revealed that most DARs are in introns (37\%), distal intergenic regions $(35 \%)$ or promoter regions $(21 \%)$ (Figure $1 \mathrm{~b}$ ). This distribution was similar to the distribution of all identified 158,534 peaks. The total number of DARs were annotated to 20,255 known genes, out of which, statistically significant DARs were annotated to 9,427 genes (FDR<0.05). In this study we focused on DARs which were mapped to the most fundamental regulatory element of a gene, its promoter region (0-3 kilobase $(\mathrm{kb})$ from annotated Transcription Start Site (TSS)). To confirm that the DARs identified here are specific to ectopic BORIS expression and not due to the presence of dox, we generated ATAC-Seq data of EV cells cultured without dox (EVneg) and compared those to the dox cultured EV cells (EVpos). These data revealed no statically significant DARs between EVneg and EVpos (Supp 5). Indicating that the reported DARs are indeed due to ectopic BORIS expression 


\subsection{Ectopic BORIS expression leads to increased chromatin accessibility at promoter regions}

To address if ectopic BORIS expression preferentially leads to a gain or loss in chromatin accessibility in MM057 melanoma cells, we determined the fold change in chromatin accessibility between BORpos and EVpos. Out of all 22,605 statistically significant DARs (FDR<0.05), 5,293 DARs had increased chromatin accessibility $(\log F C>1)$, compared to 5,347 DARs that showed reduced chromatin accessibility $(\operatorname{logFC}<-1)$. Given the role of BORIS in transcriptional regulation at promoters(Bergmaier et al., 2018), we next focused on DARs that map to gene promoter regions (0$3 \mathrm{~kb}$ around or from the annotated TSS). This analysis revealed 4,812 statistically significant DARs in promoter regions. Interestingly, $17 \%$ of these DARs showed an increase in accessibility $\left(\log _{2} \mathrm{FC}>1\right.$, $\mathrm{N}=651)$. While only $10 \%$ of DARs were downregulated $\left(\log _{2} \mathrm{FC}<-1, \mathrm{~N}=393\right)$ (Figure 2). These findings suggest that ectopic BORIS expression contributes to increased chromatin accessibility at promoters.

To gain insight into potential functional consequences of altered chromatin accessibility at promoter regions, we performed an enrichment analysis of gene ontologies biological processes (GO-BP), for significant DARs mapped to promoters (0-3kb), using the rGREAT software(McLean et al., 2010; Zuguang $\mathrm{Gu}, 2020$ ). Of particular interest is the enrichment of the biological process 'substratedependent cell migration, cell extension', as we have previously demonstrated increased cell migration upon ectopic BORIS expression (Janssen et al., 2020) (Figure 3). Also, biological processes such as stem cell differentiation and negative regulation of T-cells were found to be enriched. Together, these data suggest that increased chromatin accessibility at promoter regions may contribute to altered expression of genes involved in, amongst others, cell migration, while downregulated processes included proliferation.

\subsection{Ectopic BORIS expression leads to increased promoter accessibility in conjunction with elevated gene expression at a subset of upregulated genes.}

We set out to determine if BORIS mediated chromatin accessibility changes directly influence gene expression. Differentially Expressed Genes (DEGs) upon ectopic BORIS expression in MM057 melanoma cells were previously identified using RNA-seq(Janssen et al., 2020). As the ATAC-Seq data is obtained with the same system as the RNA-Seq, and the same differential analysis between the samples (BORpos vs EVpos), we were able to compare identified DEGs and promoter DARs mapped to their corresponding gene. To increase the chances for finding overlaps between the gene lists, we searched for genes which had the same directionality (up or downregulated) in both lists. The statistically significant $(\mathrm{FDR}<0.05)$ gene list of upregulated $(\log F C>0)$ RNA-Seq-DEGs consisted out of 1,307 genes, and the ATAC-Seq DARs contained 2,929 genes. While the downregulated gene list $(\log \mathrm{FC}<0)$ of RNA-Seq DEGs contained 935 genes and the ATAC-Seq DARs had 881 genes. 
Taken together, these intersected lists included 534 genes, which showed significant differential accessibility of promoter region, coupled with significant mRNA differential expression, in response to BORIS ectopic expression in melanoma cells (Figure 4a,b). Concordance of directionality in expression and prompter accessibility changes observed for 534 genes. Vast majority of genes 442 (83\%) were upregulated, while mere 92 (17\%) were downregulated (Fig 4a,b). As anticipated based on previous studies linking ectopic BORIS expression to melanoma(Hines, Bazarov, Mukhopadhyay, \& Yaswen, 2010; Janssen et al., 2020; Kholmanskikh, Loriot, Brasseur, De Plaen, \& De Smet, 2008), known cancer related driving genes such as PD-L1, GDF6, TNFA-IP2, IL1- $\beta$, CTLA-4, CDX2, are present among the overlapping DEGs and DARs (Supp 1.). Now that we have established a list of DARs and DEGs genes, we wanted to test for their functional enrichment GO-BP using rGREAT software (McLean et al., 2010; Zuguang Gu, 2020), has been done for the significant promoter DARs. This analysis was conducted only with the 442 genes identified as upregulated DARs \& DEGS, since the number of the downregulated genes was too small (92 genes) to obtain significant results. This subset of genes was enriched for cell migration, growth, and angiogenesis (Figure 4c). Together, these data reveal that a large subset of genes that become upregulated upon ectopic BORIS expression also gain promoter accessibility, including melanoma-promoting genes.

\subsection{ATAC-Seq DARs mapped to genes promoters show high similarity when compared to Differentially Expressed Genes (DEGs) derived from RNA-Seq data}

To fully corroborate the functional annotation of ATAC-seq identified promoter DARs, a gene set enrichment analysis (GSEA) was performed with GSEA (Subramanian et al., 2005) for gene sets representing GO-BP as well as melanoma invasive gene-set (Verfaillie et al., 2015). For this analysis, all genes with a peak mapped to their promoter were included (16,160 genes in total). The peaks were annotated to genes and pre-ranked by $\log _{2} \mathrm{FC}$ value of the resulted analysis between BORpos vs EVpos samples. Similar to the identified significant peaks defined as DARs mapped to promoters, among all peaks mapped to promoters used here, $81.5 \%$ showed increased chromatin accessibility (positive $\log _{2} \mathrm{FC}$ value) and only $18.5 \%$ showed decreased chromatin accessibility (negative $\log _{2} \mathrm{FC}$ value). We observed a strong positive enrichment for multiple biological processes involved in epithelial-mesenchymal transition, cell migration as well as the melanoma specific invasive gene signature. These data are in agreement with the observations we previously reported with a similar RNA-seq based analysis, and further support the idea that ectopic BORIS expression may promote an invasive gene signature and phenotype in melanoma cells by altering chromatin accessibility. The full concordance between the same GO-BP and melanoma invasiveness gene set results of ATAC-Seq and RNA-Seq analyses are presented on Figure 5.

\subsection{Integrated ATAC-seq and RNA-seq analysis indicates differential transcription factor activity, including of cancer-related TFs, upon ectopic BORIS expression}


To gain further insight into BORIS-mediated transcriptional regulation of the overlapping DEGs and DARs defined above, we assessed differential TF activity between BORpos vs EVpos using diffTF (Berest et al., 2019). Briefly, this is a novel multi-platform software package, specifically developed to estimate differential TFs activity based on differences in chromatin exposure around TFs DNAbinding sites, as captured by ATAC-Seq. Integration with RNA-seq gene expression, proposes which TFs acts as activators and which as repressors, bases on the differential expression of the genes they regulate.

This analysis revealed that ectopic BORIS expression regulates 70 statistically significant TFs motifs (FDR $<0.05)$; 32 activators and 38 repressors. To narrow down the TFs list to unambiguous TFs that were differentially activated or repressed upon ectopic BORIS expression, we established a list of TFs that were DOX-induced. For this purpose, another comparison was made between the controls: EVpos vs EVneg. Indicating the differential TF activity is due solely to the addition of dox. Any TF that was classified as activator or repressor with a threshold of FDR $<0.1$ in this list was subtracted from the final list of TFs. During the examination of the results, we noticed a few TFs with two significant different motifs. TFs with a double motif that were classified as activators were: MAZ, SP1 along with the ones classified as repressors: NFIA, P73, SOX10. Thus, reducing the final list of TFs differentially regulated upon ectopic BORIS expression to 24 activators and 27 repressors (Figure 6). We noticed various TFs associated with tumor progression among the TFs activated in BORIS expressing melanoma cells, such as SP1, STAT6, HOXA1, PBX3, TEAD4, NFY-B and NFY-C. Similarly, TFs known to act as tumor suppressors, including P53, P73, NFIA, TFAP2C are preferentially repressed by BORIS. Together, these findings reveal TFs that are likely differentially regulated by BORIS and suggest that BORIS-induced gene expression changed could be mediated, at least in part, by altered TF activity.

\section{DISCUSSION}

In mammalians, BORIS is the sole brother gene of CTCF, the multifunctional transcription factor. While CTCF is expressed in many tissues, BORIS functional expression is limited to the testis, and it becomes erroneously reactivated in various cancer types, including melanoma (Loukinov et al., 2002; Soltanian \& Dehghani, 2018). In our latest endeavor to study BORIS in melanoma, we sought to identify its key target genes and TFs, which drive the disease progression, with an emphasis on biological processes which are involved in the switching towards an invasiveness phenotype. Here, we uncovered some of BORIS's essential co-regulators and effectors, which exhibited differential activity in response to BORIS ectopic expression in melanocytes. We have shown this through the study of altered chromatin accessibility and mRNA expression.

In this study we were interested in BORIS target genes, that meat two criteria; first their promoter region (0-3kb) chromatin accessibility, was differentially regulated (i.e., DARs). Second, the gene's mRNA transcript was differentially expressed (i.e., DEGs), as identified in our previous research. We 
have found BORIS ectopic expression induces the differential upregulation of several tumor progressors genes, which are involve in invasive cell behavior of metastatic malignant development. Some oncogenes were classified as both DARs \& DEGs, the most notable one is PD-L1(CD274) (Akiyama, Matsuda, Arai, \& Saeki, 2020), a main negative regulator of the immune system. Another immune suppressor was CTLA4 (Chambers, Kuhns, Egen, \& Allison, 2001), which serves as an immunotherapy target in melanoma (Ribas et al., 2009). Upregulates of a few tropomyosin receptor kinase (TRK) family members was observed; neurotrophic tyrosine receptor kinase 1 (NTRK1), NTRK2, NTRK3 (Khotskaya et al., 2017), which were linked to poor survival in kidney cancer (Eggert et al., 2001) and been suggested to play an important role in mediating breast cancer brain metastatic dissemination (Bollig-Fischer et al., 2015). A key melanoma metastasis driver gene: Growth Differentiation Factor 6 (GDF6) was also at the top of the DAR \& DEG list (Figure 7). GDF6 is a ligand of the Bone morphogenetic proteins (BMPs) signaling pathway, required for tumor maintenance and was recently pointed as a key factor in melanoma tumor progression and the formation of metastasis (Venkatesan et al., 2018) as it's been reported to be amplified and transcriptionally upregulated in melanoma. GDF6 helps to maintain a trunk neural crest gene signature in melanoma. Additionally, GDF6 repressed MITF and the pro-apoptotic factor SOX9, therefore preventing differentiation, inhibiting cell death, and supporting tumor growth. Additional GDF members genes were found as DARs \& DEGs include GDF7,GDF11 and GDF15. GDF15 is a serum marker for metastases in uveal melanoma patients (Suesskind et al., 2012), in addition to its link to PD-L1 expression regulation in glioblastoma (Peng, Li, Fu, \& Zhou, 2019). GDF11 upregulation is linked to shorter overall-survival of uveal melanoma (X. Liu et al., 2019). Other members of the BMP family were found to be significant DARs \& DEGs; BMP2 and BMP6 along with BMPER (BMP endothelial cell precursor-derived regulator). BMP2 ectopic expression was identified in melanoma cell lines (Langenfeld et al., 2003) and its role in tumor process including migration and invasion, in addition to stimulating tumor growth in lung carcinomas (Rothhammer et al., 2005). BMP6 enhanced expression in melanoma promotes tumor development and growth via inhibitory effects on dermal mast cell infiltration (Stieglitz et al., 2019). BMPER is a direct extracellular BMP modulator, that is necessary for BMPs to exert their full range signaling activity. Lack of BMPER results in inhibition of tumor growth. BMPER controls features of malignant cell behavior, such as cell proliferation and migration (Heinke et al., 2012). As a BMP-binding protein, it directly interacts with BMP2, BMP4, and BMP6 (Moser et al., 2003). BMPs are members of the transforming growth factor (TGF) superfamily, TGF- $\beta$ members showed to be upregulated by BORIS in neuroblastoma (Makani et al., 2021) in addition our research team showed this in melanoma (Janssen et al., 2020).

Examining the impact of BORIS ectopic expression on the transcription regulators in melanoma cells revealed some TFs linked to the advance stages of the disease. This analysis was conducted with 
DiffTF (Berest et al., 2019), a novel multi-platform tool combining ATAC-Seq accessible DNA regions with RNA-Seq gene expression, for the classification of TFs as activators or repressors, based on differential comparison between the tested condition (BORpos) to the control (EVpos). We noticed a trend for tumor driver to classify as activated TFs, while tumor suppressors were classified as TFs with repressed activity. The AP-1/TEAD families members were identified as master regulators of the invasive gene network (Verfaillie et al., 2015), here we have identified a few family members to be differentially upregulated and classified as activators TFs (Figure 6). A critical member is TEAD4, which was linked to genetic signature of EMT and invasiveness, resulting in aggressiveness and poor prognosis in head neck squamous cell carcinoma (W. Zhang et al., 2018). Furthermore, SP-1 a member of the Sp-family (Beishline \& Azizkhan-Clifford, 2015; Vizcaíno, Mansilla, \& Portugal, 2015), which were suggested to play a pivotal role in tumor progression and metastasis, was found to be differentially activated. Along with it, MAZ (Maity et al., 2018) was upregulated, it has been identified as a binding factor to Sp-family promoters. STAT6 a cancer growth factor, which was found to be required for EMT or to promote invasive growth and metastatic niche formation (Binnemars-Postma, Bansal, Storm, \& Prakash, 2018; Chen et al., 2018; Leon-Cabrera et al., 2017; Lu, Shi, \& Zheng, 2018; Merk, Owens, Lopes, Silva, \& Hussaini, 2011), was also identified here as an activated TF. Other tumor progressors TFs were classified as activators, among them HOXA1 a melanoma driver tumor growth that evokes invasion gene expression signature leading to metastasis (Wardwell-Ozgo et al., 2014). The Nuclear factor of activated T cells (NFAT) (Flockhart, Armstrong, Reynolds, \& Lovat, 2009) members NFATC4, which supports melanoma cell transformation and tumor growth (Xiao et al., 2017) in addition to the activation of another member - NFATC3. The NFAT-family members were shown to be activated by oncogenic BRAF in melanoma, our findings show a similar role of BORIS ectopic expression in melanoma, as it can activate the same pathways. In our current study we have linked TGF- $\beta$ targets such as NGFR and JUNB, which are regulators of melanoma phenotype switching (Restivo et al., 2017; Verfaillie et al., 2015), to BORIS ectopic expression in melanocytes as those genes were defined as DARs \& DEGs. Some melanoma related factors were found to be already activated in the EV samples and not significantly differentially regulated by BORIS ectopic expression. This can be seen when examining TFs that have been shown to be active in melanoma phenotypic switching from proliferative to invasive (Cohen-Solal, Kaufman, \& Lasfar, 2018), such as AP-1 transcription factor subunits FOSB, FOSL1, JUND, c-JUN and JUNB were found to be activators in the EV control samples, we could not clearly state those factors as differentially regulated here. Even though JUNB activity as a TF was not found to be significantly differential, it was still defined as one of BORIS target genes in the DARs \& DEGs defined gene-list. All these evidence, link numerous cancer markers genes and TFs, which drive the cancerous processes to its advance stages, to BORIS ectopic expression in melanoma. When pointing at tumor suppressors factors, the TFs SOX10/MITF were identified as master regulators of the proliferative gene network. In our analyses we found SOX10 to be differentially repressed, as it is classified as a 
repressor, unique to BORpos condition. An invasion preventor gene MTSS1 (Vadakekolathu et al., 2018), was among the genes that were differentially downregulated both as DAR \& DEG. Also downregulated is the cancer-testis antigen Rhophilin-associated tail protein 1 (ROPN1), it is a part of several conserved elements of the SOX10 signature(Ivanov et al., 2013), which was found to be repressed here. Some crucial tumor suppressors TFs, were found to be differentially represses, among them TP53 and its related P73 and PATZ1 which upregulates TP53 related genes (Valentino et al., 2013), in addition to the downregulation of MAGED1(NRAGE), which upregulates TP53 and suppresses cellular growth and invasion(Du, Zhang, Tian, Li, \& Fang, 2009). A controversial TF in cancer - NFAT5(Jiang et al., 2019) was classified as a repressor, while other NFAT family members were activated, indicating it might serve as a tumor suppressor in melanocytes. Other factors such as STAT3 and c-Myc activity in the BORpos samples was classified as undetermined. The downregulated factors were few, but they included some notable tumor suppressors, which strengthen the assumption that BORIS ectopic expression might contribute to the regulation of the cancerous development processes with a depressive effect on tumor suppressors factors, thus enabling tumor driving genes to dominate.

Enrichment analysis of DARs mapped to promoters $(0-3 \mathrm{~kb})$ revealed their involvement in some melanoma progression processes. When comparing to our previous enrichment analysis based on RNA-Seq DEGs, the same biological processes were found to be enriched both at the DNA-promoter level and mRNA transcript level. Such processes include EMT, cell motility and migration, taxis, locomotion and invasiveness signature (Figure 5). These findings show BORIS' capabilities to modify chromatin accessibility that directly influences gene expression, turning the transcriptional landscape of the cell en route to invade surrounding tissue.

\section{Summary}

Here we presented a combined analysis of ATAC-Seq and RNA-Seq aimed at exploring the impact of BORIS ectopic expression on chromatin accessibility and gene expression in melanoma cells. Chromatin differential accessibility analysis revealed which genes promoter's regions ( $0-3 \mathrm{~kb}$ from annotated TSS) chanced accessibility in a differential manner. Enrichment analysis of biological processes identified many pro-invasiveness pathways as significant. The intersect of ATAC-Seq and RNA-Seq, generated the suspected BORIS target genes, that are differentially regulated by BORIS at the gene's promoter's level, as well as the mRNA transcript level. Among the upregulated BORIS targets, immune inhibitors such as PD-L1 and CTLA4 in addition to GDF6 a novel metastasis driver gene. Moreover, differential TFs activity analysis revealed BORIS activated tumor progressor TFs such as TEAD4, STAT6, NFYB and AP-1 family, while differentially repressed pro-proliferative and tumor suppressors TFs such as SOX10, P53, P73, MAGED1. From our findings of chromatin accessibility and gene expression we increase the evidence situating BORIS as a key regulator of tumor invasiveness in melanoma and progression of the disease. In our study we did not explore 
genomic enhancers or protein level control, such as post translational modifications (PTM). We cannot rule out the possibility of an effect in our results, that could arise from unexamined cellular regulation machinery. Nevertheless, BORIS should be considered as an important target in melanoma, as it may serve a possible candidate for future immunotherapies, such as anti-BORIS vaccination, in patients with high BORIS levels. Further research of clinical data from melanoma patients, is needed to test the evidence reported here. This study can serve as a basis for classifying melanoma patients according to BORIS activity level, by identifying not only BORIS expression, but also the unique pattern of genetic alterations it creates. Specifically, BORIS ectopic expression differentially activated and repressed unique TFs and genes in melanoma cells, as presented here. By utilizing this approach, we can single out possible anti-BORIS vaccine candidates, based not only on BORIS expression level but also on BORIS measured activity level in melanoma. By doing so we could define a BORIS signature that may be melanoma specific or pan cancer relevant. 


\section{Reference}

Akiyama, M., Matsuda, Y., Arai, T., \& Saeki, H. (2020). PD-L1 expression in malignant melanomas of the skin and gastrointestinal tract. Oncology Letters, 19(3), 2481-2488. https://doi.org/10.3892/ol.2020.11325

Ambrosini, G., Groux, R., \& Bucher, P. (2018). PWMScan: a fast tool for scanning entire genomes with a position-specific weight matrix. Bioinformatics (Oxford, England), 34(14), 2483-2484. https://doi.org/10.1093/bioinformatics/bty127

Andrews, S., Krueger, F., Seconds-Pichon, A., Biggins, F., \& Wingett, S. (2010). FastQC. A quality control tool for high throughput sequence data. Babraham Bioinformatics. Babraham Institute, 1(1), 1. Retrieved from https://www.bioinformatics.babraham.ac.uk/projects/fastqc/\%0Ahttp://www.bioinformatics.bbsr c.ac.uk/projects/fastqc/

Beishline, K., \& Azizkhan-Clifford, J. (2015). Sp1 and the "hallmarks of cancer." FEBS Journal, 282(2), 224-258. https://doi.org/10.1111/febs.13148

Benjamini, Y., \& Hochberg, Y. (1995). Controlling the false discovery rate: a practical and powerful approach to multiple testing. Journal of the Royal Statistical Society, Vol. 57, pp. 289-300. https://doi.org/10.2307/2346101

Berest, I., Arnold, C., Reyes-Palomares, A., Palla, G., Rasmussen, K. D., Helin, K., \& Zaugg, J. (2019). Quantification of Differential Transcription Factor Activity and Multiomics-Based Classification into Activators and Repressors: DiffTF. SSRN Electronic Journal, 29(10), 31473159.e12. https://doi.org/10.2139/ssrn.3329887

Bergmaier, P., Weth, O., Dienstbach, S., Boettger, T., Galjart, N., Mernberger, M., ... Renkawitz, R. (2018). Choice of binding sites for CTCFL compared to CTCF is driven by chromatin and by sequence preference. Nucleic Acids Research, 46(14), 7097-7107. https://doi.org/10.1093/nar/gky483

Binnemars-Postma, K., Bansal, R., Storm, G., \& Prakash, J. (2018). Targeting the Stat6 pathway in tumor-associated macrophages reduces tumor growth and metastatic niche formation in breast cancer. FASEB Journal, 32(2), 969-978. https://doi.org/10.1096/fj.201700629R

Bollig-Fischer, A., Michelhaugh, S. K., Wijesinghe, P., Dyson, G., Kruger, A., Palanisamy, N., ... Mittal, S. (2015). Cytogenomic profiling of breast cancer brain metastases reveals potential for repurposing targeted therapeutics. Oncotarget, 6(16), 14614-14624. https://doi.org/10.18632/oncotarget.3786 
Buenrostro, J. D., Giresi, P. G., Zaba, L. C., Chang, H. Y., \& Greenleaf, W. J. (2013). Transposition of native chromatin for fast and sensitive epigenomic profiling of open chromatin, DNA-binding proteins and nucleosome position. Nature Methods, 10(12), 1213-1218. https://doi.org/10.1038/nmeth.2688

Bushnell B. (2014). BBMap short-read aligner, and other bioinformatics tools. Retrieved October 8 , 2018, from https://sourceforge.net/projects/bbmap/

Chambers, C. A., Kuhns, M. S., Egen, J. G., \& Allison, J. P. (2001). CTLA-4-mediated inhibition in regulation of $\mathrm{T}$ cell responses: Mechanisms and manipulation in tumor immunotherapy. Annual Review of Immunology, Vol. 19, pp. 565-594. https://doi.org/10.1146/annurev.immunol.19.1.565

Cheema, Z., Hari-Gupta, Y., Kita, G. X., Farrar, D., Seddon, I., Corr, J., \& Klenova, E. (2014). Expression of the cancer-testis antigen BORIS correlates with prostate cancer. Prostate, 74(2), 164-176. https://doi.org/10.1002/pros.22738

Chen, J., Gong, C., Mao, H., Li, Z., Fang, Z., Chen, Q., ... Li, H. (2018). E2F1/SP3/STAT6 axis is required for IL-4-induced epithelial-mesenchymal transition of colorectal cancer cells. International Journal of Oncology, 53(2), 567-578. https://doi.org/10.3892/ijo.2018.4429

Cohen-Solal, K. A., Kaufman, H. L., \& Lasfar, A. (2018). Transcription factors as critical players in melanoma invasiveness, drug resistance, and opportunities for therapeutic drug development. Pigment Cell and Melanoma Research, 31(2), 241-252. https://doi.org/10.1111/pcmr.12666

D’Arcy, V., Pore, N., Docquier, F., Abdullaev, Z. K., Chernukhin, I., Kita, G. X., ... Klenova, E. (2008). BORIS, a paralogue of the transcription factor, CTCF, is aberrantly expressed in breast tumours. British Journal of Cancer, 98(3), 571-579. https://doi.org/10.1038/sj.bjc.6604181

Dahl, C., \& Guldberg, P. (2007). The genome and epigenome of malignant melanoma. Apmis, 115(10), 1161-1176. https://doi.org/10.1111/j.1600-0463.2007.apm_855.xml.x

Dougherty, C. J., Ichim, T. E., Liu, L., Reznik, G., Min, W. P., Ghochikyan, A., ... Reznik, B. N. (2008). Selective apoptosis of breast cancer cells by siRNA targeting of BORIS. Biochemical and Biophysical Research Communications, 370(1), 109-112. https://doi.org/10.1016/j.bbrc.2008.03.040

Du, Q., Zhang, Y., Tian, X.-X. X., Li, Y., \& Fang, W.-G. G. (2009). MAGE-D1 inhibits proliferation, migration and invasion of human breast cancer cells. Oncology Reports, 22(3), 659-665. https://doi.org/10.3892/or_00000486

Eggert, A., Grotzer, M. A., Ikegaki, N., Zhao, H., Cnaan, A., Brodeur, G. M., \& Evans, A. E. (2001). 
Expression of the neurotrophin receptor TrkB is associated with unfavorable outcome in Wilms' tumor. Journal of Clinical Oncology, 19(3), 689-696.

https://doi.org/10.1200/JCO.2001.19.3.689

Fane, M. E., Chhabra, Y., Hollingsworth, D. E. J., Simmons, J. L., Spoerri, L., Oh, T. G., ... Smith, A. G. (2017). NFIB Mediates BRN2 Driven Melanoma Cell Migration and Invasion Through Regulation of EZH2 and MITF. EBioMedicine, 16, 63-75. https://doi.org/10.1016/j.ebiom.2017.01.013

Feng, J., Liu, T., Qin, B., Zhang, Y., \& Liu, X. S. (2012). Identifying ChIP-seq enrichment using MACS. Nature Protocols, 7(9), 1728-1740. https://doi.org/10.1038/nprot.2012.101

Fiziev, P., Akdemir, K. C., Miller, J. P., Keung, E. Z., Samant, N. S., Sharma, S., .. Rai, K. (2017). Systematic Epigenomic Analysis Reveals Chromatin States Associated with Melanoma Progression. Cell Reports, 19(4), 875-889. https://doi.org/10.1016/j.celrep.2017.03.078

Flockhart, R. J., Armstrong, J. L., Reynolds, N. J., \& Lovat, P. E. (2009). NFAT signalling is a novel target of oncogenic BRAF in metastatic melanoma. British Journal of Cancer, 101(8), 14481455. https://doi.org/10.1038/sj.bjc.6605277

Gaykalova, D., Vatapalli, R., Glazer, C. A., Bhan, S., Shao, C., Sidransky, D., ... Califano, J. A. (2012). Dose-dependent activation of putative oncogene SBSN by BORIS. PLoS ONE, 7(7), e40389. https://doi.org/10.1371/journal.pone.0040389

He, S. J., Stevens, G., Braithwaite, A. W., \& Eccles, M. R. (2005). Transfection of melanoma cells with antisense PAX3 oligonucleotides additively complements cisplatin-induced cytotoxicity. Molecular Cancer Therapeutics, 4(6), 996-1003. https://doi.org/10.1158/1535-7163.MCT-040252

Heinke, J., Kerber, M., Rahner, S., Mnich, L., Lassmann, S., Helbing, T., .. Moser, M. (2012). Bone morphogenetic protein modulator BMPER is highly expressed in malignant tumors and controls invasive cell behavior. Oncogene, 31(24), 2919-2930. https://doi.org/10.1038/onc.2011.473

Hillman, J. C., Pugacheva, E. M., Barger, C. J., Sribenja, S., Rosario, S., Albahrani, M., ... Karpf, A. R. (2019). BORIS expression in ovarian cancer precursor cells alters the CTCF cistrome and enhances invasiveness through GALNT14. Molecular Cancer Research, 17(10), 2051-2062. https://doi.org/10.1158/1541-7786.MCR-19-0310

Hines, W. C., Bazarov, A. V, Mukhopadhyay, R., \& Yaswen, P. (2010). BORIS (CTCFL) is not expressed in most human breast cell lines and high grade breast carcinomas. PLOS ONE, 5(3), e9738. https://doi.org/10.1371/journal.pone.0009738 
Hoek, K. S., Eichhoff, O. M., Schlegel, N. C., Döbbeling, U., Kobert, N., Schaerer, L., ... Dummer, R. (2008). In vivo switching of human melanoma cells between proliferative and invasive states. Cancer Research, 68(3), 650-656. https://doi.org/10.1158/0008-5472.CAN-07-2491

Hoek, K. S., \& Goding, C. R. (2010, December). Cancer stem cells versus phenotype-switching in melanoma. Pigment Cell and Melanoma Research, Vol. 23, pp. 746-759. https://doi.org/10.1111/j.1755-148X.2010.00757.x

Hoek, K. S., Schlegel, N. C., Brafford, P., Sucker, A., Ugurel, S., Kumar, R., ... Dummer, R. (2006). Metastatic potential of melanomas defined by specific gene expression profiles with no BRAF signature. Pigment Cell Research, 19(4), 290-302. https://doi.org/10.1111/j.16000749.2006.00322.x

Hoek, K. S., Schlegel, N. C., Eichhoff, O. M., Widmer, D. S., Praetorius, C., Einarsson, S. O., ... Steingrimsson, E. (2008). Novel MITF targets identified using a two-step DNA microarray strategy. Pigment Cell and Melanoma Research, 21(6), 665-676. https://doi.org/10.1111/j.1755148X.2008.00505.x

Huber, W., Carey, V. J., Gentleman, R., Anders, S., Carlson, M., Carvalho, B. S., ... Morgan, M. (2015). Orchestrating high-throughput genomic analysis with Bioconductor. Nature Methods, 12(2), 115-121. https://doi.org/10.1038/nmeth.3252

Ivanov, S. V, Panaccione, A., Nonaka, D., Prasad, M. L., Boyd, K. L., Brown, B., ... Yarbrough, W. G. (2013). Diagnostic SOX10 gene signatures in salivary adenoid cystic and breast basal-like carcinomas. British Journal of Cancer, 109(2), 444-451. https://doi.org/10.1038/bjc.2013.326

Janssen, S. M., Moscona, R., Elchebly, M., Papadakis, A. I., Redpath, M., Wang, H., ... Spatz, A. (2020). BORIS/CTCFL promotes a switch from a proliferative towards an invasive phenotype in melanoma cells. Cell Death Discovery 2020 6:1, 6(1), 1-17. https://doi.org/10.1038/s41420019-0235-x

Jeffs, A. R., Glover, A. C., Slobbe, L. J., Wang, L., He, S., Hazlett, J. A., ... Eccles, M. R. (2009). A gene expression signature of invasive potential in metastatic melanoma cells. PLoS ONE, 4(12). https://doi.org/10.1371/journal.pone.0008461

Jiang, Y., He, R., Jiang, Y., Liu, D., Tao, L., Yang, M., .. Sun, Y. (2019). Transcription factor NFAT5 contributes to the glycolytic phenotype rewiring and pancreatic cancer progression via transcription of PGK1. Cell Death and Disease, 10(12), 1-13. https://doi.org/10.1038/s41419019-2072-5

Jochum, W., Passegué, E., \& Wagner, E. F. (2001, April 30). AP-1 in mouse development and tumorigenesis. Oncogene, Vol. 20, pp. 2401-2412. https://doi.org/10.1038/sj.onc.1204389 
Kemp, C. J., Moore, J. M., Moser, R., Bernard, B., Teater, M., Smith, L. E., ... Filippova, G. N. (2014). CTCF haploinsufficiency destabilizes DNA methylation and predisposes to cancer. Cell Reports, 7(4), 1020-1029. https://doi.org/10.1016/j.celrep.2014.04.004

Kholmanskikh, O., Loriot, A., Brasseur, F., De Plaen, E., \& De Smet, C. (2008). Expression of BORIS in melanoma: Lack of association with MAGE-A1 activation. International Journal of Cancer, 122(4), 777-784. https://doi.org/10.1002/ijc.23140

Khotskaya, Y. B., Holla, V. R., Farago, A. F., Mills Shaw, K. R., Meric-Bernstam, F., \& Hong, D. S. (2017, May 1). Targeting TRK family proteins in cancer. Pharmacology and Therapeutics, Vol. 173, pp. 58-66. https://doi.org/10.1016/j.pharmthera.2017.02.006

Klenova, E. M., Morse, H. C., Ohlsson, R., \& Lobanenkov, V. V. (2002). The novel BORIS + CTCF gene family is uniquely involved in the epigenetics of normal biology and cancer. Seminars in Cancer Biology, 12(5), 399-414. https://doi.org/10.1016/S1044-579X(02)00060-3

Kulakovskiy, I. V., Vorontsov, I. E., Yevshin, I. S., Sharipov, R. N., Fedorova, A. D., Rumynskiy, E. I., ... Makeev, V. J. (2018). HOCOMOCO: Towards a complete collection of transcription factor binding models for human and mouse via large-scale ChIP-Seq analysis. Nucleic Acids Research, 46(D1), D252-D259. https://doi.org/10.1093/nar/gkx1106

Langenfeld, E. M., Calvano, S. E., Abou-Nukta, F., Lowry, S. F., Amenta, P., \& Langenfeld, J. (2003, September 1). The mature bone morphogenetic protein-2 is aberrantly expressed in non-small cell lung carcinomas and stimulates tumor growth of A549 cells. Carcinogenesis, Vol. 24, pp. 1445-1454. https://doi.org/10.1093/carcin/bgg100

Leon-Cabrera, S. A., Molina-Guzman, E., Delgado-Ramirez, Y. G., Vázquez-Sandoval, A., LedesmaSoto, Y., Pérez-Plasencia, C. G., ... Terrazas, L. I. (2017). Lack of STAT6 attenuates inflammation and drives protection against early steps of colitis-associated colon cancer. Cancer Immunology Research, 5(5), 385-396. https://doi.org/10.1158/2326-6066.CIR-16-0168

Li, H. (2013). Aligning sequence reads, clone sequences and assembly contigs with BWA-MEM. Genome Biology, 15(12), 1-3. https://doi.org/arXiv:1303.3997 [q-bio.GN]

Li, H., Handsaker, B., Wysoker, A., Fennell, T., Ruan, J., Homer, N., .. 1000 Genome Project Data Processing Subgroup. (2009). The Sequence Alignment/Map format and SAMtools.

Bioinformatics, 25(16), 2078-2079. https://doi.org/10.1093/bioinformatics/btp352

Liu, Q., Chen, K., Liu, Z., Huang, Y., Zhao, R., Wei, L., ... Li, B. (2017). BORIS up-regulates OCT4 via histone methylation to promote cancer stem cell-like properties in human liver cancer cells. Cancer Letters, 403, 165-174. https://doi.org/10.1016/j.canlet.2017.06.017 
Liu, X., Zhang, Q., Fan, C., Tian, J., Liu, X., \& Li, G. (2019). GDF11 upregulation independently predicts shorter overall-survival of uveal melanoma. PLOS ONE, 14(3), e0214073. https://doi.org/10.1371/journal.pone.0214073

Loukinov, D. I., Pugacheva, E., Vatolin, S., Pack, S. D., Moon, H., Chernukhin, I., ... Lobanenkov, V. V. (2002). BORIS, a novel male germ-line-specific protein associated with epigenetic reprogramming events, shares the same 11-zinc-finger domain with $\mathrm{CTCF}$, the insulator protein involved in reading imprinting marks in the soma. Proceedings of the National Academy of Sciences of the United States of America, 99(10), 6806-6811. https://doi.org/10.1073/pnas.092123699

Love, M. I., Huber, W., \& Anders, S. (2014). Moderated estimation of fold change and dispersion for RNA-seq data with DESeq2. Genome Biology, 15(12), 550. https://doi.org/10.1186/s13059-0140550-8

Lu, G., Shi, W., \& Zheng, H. (2018). Inhibition of STAT6/Anoctamin-1 Activation Suppresses Proliferation and Invasion of Gastric Cancer Cells. Cancer Biotherapy and Radiopharmaceuticals, 33(1), 3-7. https://doi.org/10.1089/cbr.2017.2287

Maity, G., Haque, I., Ghosh, A., Dhar, G., Gupta, V., Sarkar, S., .. Banerjee, S. (2018). The MAZ transcription factor is a downstream target of the oncoprotein Cyr61/CCN1 and promotes pancreatic cancer cell invasion via CRAF-ERK signaling. Journal of Biological Chemistry, 293(12), 4334-4349. https://doi.org/10.1074/jbc.RA117.000333

Makani, V. K. K., Mendonza, J. J., Edathara, P. M., Yerramsetty, S., \& Pal Bhadra, M. (2021). BORIS/CTCFL expression activates the TGF $\beta$ signaling cascade and induces Drp1 mediated mitochondrial fission in neuroblastoma. Free Radical Biology and Medicine, 176, 62-72. https://doi.org/10.1016/j.freeradbiomed.2021.09.010

Martin, M. (2011). Cutadapt removes adapter sequences from high-throughput sequencing reads. EMBnet.Journal, 17(1), 10. https://doi.org/10.14806/ej.17.1.200

McLean, C. Y., Bristor, D., Hiller, M., Clarke, S. L., Schaar, B. T., Lowe, C. B., .. Bejerano, G. (2010). GREAT improves functional interpretation of cis-regulatory regions. Nature Biotechnology, 28(5), 495-501. https://doi.org/10.1038/nbt.1630

Meerbrey, K. L., Hu, G., Kessler, J. D., Roarty, K., Li, M. Z., Fang, J. E., ... Elledge, S. J. (2011). The pINDUCER lentiviral toolkit for inducible RNA interference in vitro and in vivo. Proceedings of the National Academy of Sciences of the United States of America, 108(9), 36653670. https://doi.org/10.1073/pnas.1019736108

Merk, B. C., Owens, J. L., Lopes, M. B. S., Silva, C. M., \& Hussaini, I. M. (2011). STAT6 expression 
in glioblastoma promotes invasive growth. BMC Cancer, 11(1), 184.

https://doi.org/10.1186/1471-2407-11-184

Moser, M., Binder, O., Wu, Y., Aitsebaomo, J., Ren, R., Bode, C., ... Patterson, C. (2003). BMPER, a Novel Endothelial Cell Precursor-Derived Protein, Antagonizes Bone Morphogenetic Protein Signaling and Endothelial Cell Differentiation. Molecular and Cellular Biology, 23(16), 5664 5679. https://doi.org/10.1128/mcb.23.16.5664-5679.2003

Parker, S. (2015). atactk: a toolkit for ATAC-seq data - atactk 0.1.5. Retrieved October 8, 2018, from https://github.com/ParkerLab/atactk/

Peng, H., Li, Z., Fu, J., \& Zhou, R. (2019). Growth and differentiation factor 15 regulates PD-L1 expression in glioblastoma. Cancer Management and Research, 11, 2653-2661. https://doi.org/10.2147/CMAR.S192095

Perotti, V., Baldassari, P., Molla, A., Nicolini, G., Bersani, I., Grazia, G., .. Mortarini, R. (2019). An actionable axis linking NFATc2 to EZH2 controls the EMT-like program of melanoma cells. Oncogene, 38(22), 4384-4396. https://doi.org/10.1038/s41388-019-0729-2

Phillips, J. E., \& Corces, V. G. (2009, June 26). CTCF: Master Weaver of the Genome. Cell, Vol. 137, pp. 1194-1211. https://doi.org/10.1016/j.cell.2009.06.001

Pinner, S., Jordan, P., Sharrock, K., Bazley, L., Collinson, L., Marais, R., ... Sahai, E. (2009). Intravital imaging reveals transient changes in pigment production and Brn2 expression during metastatic melanoma dissemination. Cancer Research, 69(20), 7969-7977. https://doi.org/10.1158/0008-5472.CAN-09-0781

Pugacheva, E. M., Suzuki, T., Pack, S. D., Kosaka-Suzuki, N., Yoon, J., Vostrov, A. A., ... Lobanenkov, V. (2010). The Structural Complexity of the Human BORIS Gene in Gametogenesis and Cancer. PLOS ONE, 5(11), e13872. https://doi.org/10.1371/JOURNAL.PONE.0013872

R Core Team. (2014). $R$ : A language and environment for statistical computing. $R$ Foundation for Statistical Computing. Retrieved from http://www.r-project.org/.

Ramírez, F., Ryan, D. P., Grüning, B., Bhardwaj, V., Kilpert, F., Richter, A. S., ... Manke, T. (2016). deepTools2: a next generation web server for deep-sequencing data analysis. Nucleic Acids Research, 44(W1), W160-W165. https://doi.org/10.1093/nar/gkw257

Rasko, J. E. J., Klenova, E. M., Leon, J., Filippova, G. N., Loukinov, D. I., Vatolin, S., ... Lobanenkov, V. V. (2001). Cell growth inhibition by the multifunctional multivalent zinc-finger factor CTCF. Cancer Research, 61(16), 6002-6007. 
Renaud, S., Loukinov, D., Alberti, L., Vostrov, A., Kwon, Y. W., Bosman, F. T., .. Benhattar, J. (2011). BORIS/CTCFL-mediated transcriptional regulation of the hTERT telomerase gene in testicular and ovarian tumor cells. Nucleic Acids Research, 39(3), 862-873. https://doi.org/10.1093/nar/gkq827

Restivo, G., Diener, J., Cheng, P. F., Kiowski, G., Bonalli, M., Biedermann, T., ... Sommer, L. (2017). Low Neurotrophin receptor CD271 regulates phenotype switching in Melanoma. Nature Communications, 8(1), 1-16. https://doi.org/10.1038/s41467-017-01573-6

Ribas, A., Comin-Anduix, B., Chmielowski, B., Jalil, J., De La Rocha, P., McCannel, T. A., ... Gomez-Navarro, J. (2009). Dendritic cell vaccination combined with CTLA4 blockade in patients with metastatic melanoma. Clinical Cancer Research, 15(19), 6267-6276. https://doi.org/10.1158/1078-0432.CCR-09-1254

Rothhammer, T., Poser, I., Soncin, F., Bataille, F., Moser, M., \& Bosserhoff, A. K. (2005). Bone morphogenic proteins are overexpressed in malignant melanoma and promote cell invasion and migration. Cancer Research, 65(2), 448-456.

Schep, A. N., Buenrostro, J. D., Denny, S. K., Schwartz, K., Sherlock, G., \& Greenleaf, W. J. (2015). Structured nucleosome fingerprints enable high-resolution mapping of chromatin architecture within regulatory regions. Genome Research, 25(11), 1757-1770. https://doi.org/10.1101/gr.192294.115

Shakhova, O., Zingg, D., Schaefer, S. M., Hari, L., Civenni, G., Blunschi, J., ... Sommer, L. (2012). Sox 10 promotes the formation and maintenance of giant congenital naevi and melanoma. Nature Cell Biology, 14(8), 882-889. https://doi.org/10.1038/ncb2535

Siegel, R. L., Miller, K. D., \& Jemal, A. (2020). Cancer statistics, 2020. CA: A Cancer Journal for Clinicians, 70(1), 7-30. https://doi.org/10.3322/caac.21590

Smith, I. M., Glazer, C. A., Mithani, S. K., Ochs, M. F., Sun, W., Bhan, S., .. Califano, J. (2009). Coordinated activation of candidate proto-oncogenes and cancer testes antigens via promoter demethylation in head and neck cancer and lung cancer. PloS One, 4(3), e4961. https://doi.org/10.1371/journal.pone.0004961

Soltanian, S., \& Dehghani, H. (2018, October 5). BORIS: A key regulator of cancer stemness. Cancer Cell International, Vol. 18, p. 154. https://doi.org/10.1186/s12935-018-0650-8

Stieglitz, D., Lamm, S., Braig, S., Feuerer, L., Kuphal, S., Dietrich, P., ... Bosserhoff, A. K. (2019). BMP6-induced modulation of the tumor micro-milieu. Oncogene, 38(5), 609-621. https://doi.org/10.1038/s41388-018-0475-x 
Subramanian, A., Tamayo, P., Mootha, V. K., Mukherjee, S., Ebert, B. L., Gillette, M. A., ... Mesirov, J. P. (2005). Gene set enrichment analysis: A knowledge-based approach for interpreting genome-wide expression profiles. Proceedings of the National Academy of Sciences, 102(43), 15545-15550. https://doi.org/10.1073/pnas.0506580102

Suesskind, D., Schatz, A., Schnichels, S., Coupland, S. E., Lake, S. L., Wissinger, B., ... HenkeFahle, S. (2012). GDF-15: A novel serum marker for metastases in uveal melanoma patients. Graefe's Archive for Clinical and Experimental Ophthalmology, 250(6), 887-895. https://doi.org/10.1007/s00417-011-1786-6

Tirosh, I., Izar, B., Prakadan, S. M., Wadsworth, M. H., Treacy, D., Trombetta, J. J., ... Garraway, L. A. (2016). Dissecting the multicellular ecosystem of metastatic melanoma by single-cell RNAseq. Science, 352(6282), 189-196. https://doi.org/10.1126/science.aad0501

Vadakekolathu, J., Al-Juboori, S. I. K., Johnson, C., Schneider, A., Buczek, M. E., Di Biase, A., ... Regad, T. (2018). MTSS1 and SCAMP1 cooperate to prevent invasion in breast cancer. Cell Death and Disease, 9(3). https://doi.org/10.1038/s41419-018-0364-9

Valentino, T., Palmieri, D., Vitiello, M., Pierantoni, G. M., Fusco, A., \& Fedele, M. (2013). PATZ1 interacts with p53 and regulates expression of p53-target genes enhancing apoptosis or cell survival based on the cellular context. Cell Death and Disease, 4(12), 1-8. https://doi.org/10.1038/cddis.2013.500

Venkatesan, A. M., Vyas, R., Gramann, A. K., Dresser, K., Gujja, S., Bhatnagar, S., ... Ceol, C. J. (2018). Ligand-activated BMP signaling inhibits cell differentiation and death to promote melanoma. Journal of Clinical Investigation, 128(1), 294-308. https://doi.org/10.1172/JCI92513

Verfaillie, A., Imrichova, H., Atak, Z. K., Dewaele, M., Rambow, F., Hulselmans, G., ... Aerts, S. (2015). Decoding the regulatory landscape of melanoma reveals TEADS as regulators of the invasive cell state. Nature Communications, 6, 1-16. https://doi.org/10.1038/ncomms7683

Vizcaíno, C., Mansilla, S., \& Portugal, J. (2015). Sp1 transcription factor: A long-standing target in cancer chemotherapy. Pharmacology and Therapeutics, 152, 111-124. https://doi.org/10.1016/j.pharmthera.2015.05.008

Wang, C., Gu, Y., Zhang, K., Xie, K., Zhu, M., Dai, N., ... Hu, Z. (2016). Systematic identification of genes with a cancer-testis expression pattern in 19 cancer types. Nature Communications, 7 , 10499. https://doi.org/10.1038/ncomms10499

Wardwell-Ozgo, J., Dogruluk, T., Gifford, A., Zhang, Y., Heffernan, T. P., Van Doorn, R., ... Scott, K. L. (2014). HOXA1 drives melanoma tumor growth and metastasis and elicits an invasion gene expression signature that prognosticates clinical outcome. Oncogene, 33(8), 1017-1026. 
https://doi.org/10.1038/onc.2013.30

Widmer, D. S., Cheng, P. F., Eichhoff, O. M., Belloni, B. C., Zipser, M. C., Schlegel, N. C., ... Hoek, K. S. (2012). Systematic classification of melanoma cells by phenotype-specific gene expression mapping. Pigment Cell and Melanoma Research, 25(3), 343-353.

https://doi.org/10.1111/j.1755-148X.2012.00986.x

Wysoker, A., Tibbetts, K., \& Fennell, T. (2009). Picard tools version 2.17.10.

Http://Broadinstitute.Github.Io/Picard/.

Xiao, T., Zhu, J. J., Huang, S., Peng, C., He, S., Du, J., ... Zheng, D. (2017). Phosphorylation of NFAT3 by CDK3 induces cell transformation and promotes tumor growth in skin cancer.

Oncogene, 36(20), 2835-2845. https://doi.org/10.1038/onc.2016.434

Yu, G., Wang, L. G., \& He, Q. Y. (2015). ChIP seeker: An R/Bioconductor package for ChIP peak annotation, comparison and visualization. Bioinformatics, 31(14), 2382-2383. https://doi.org/10.1093/bioinformatics/btv145

Zhang, W., Li, J., Wu, Y., Ge, H., Song, Y., Wang, D., ... Cheng, J. (2018). TEAD4 overexpression promotes epithelial-mesenchymal transition and associates with aggressiveness and adverse prognosis in head neck squamous cell carcinoma. Cancer Cell International, 18(1), 178. https://doi.org/10.1186/s12935-018-0675-z

Zhang, Yanmei, Fang, M., Song, Y., Ren, J., Fang, J., \& Wang, X. (2017). Brother of Regulator of Imprinted Sites (BORIS) suppresses apoptosis in colorectal cancer. Scientific Reports, 7, 40786. https://doi.org/10.1038/srep40786

Zhang, Yong, Liu, T., Meyer, C. A., Eeckhoute, J., Johnson, D. S., Bernstein, B. E., ... Liu, X. S. (2008). Model-based analysis of ChIP-Seq (MACS). Genome Biology, 9(9), R137. https://doi.org/10.1186/gb-2008-9-9-r137

Zuguang Gu. (2020). rGREAT: Client for GREAT Analysis (p. https://github.com/jokergoo/rGREAT,http://great.st). p. https://github.com/jokergoo/rGREAT,http://great.st. 


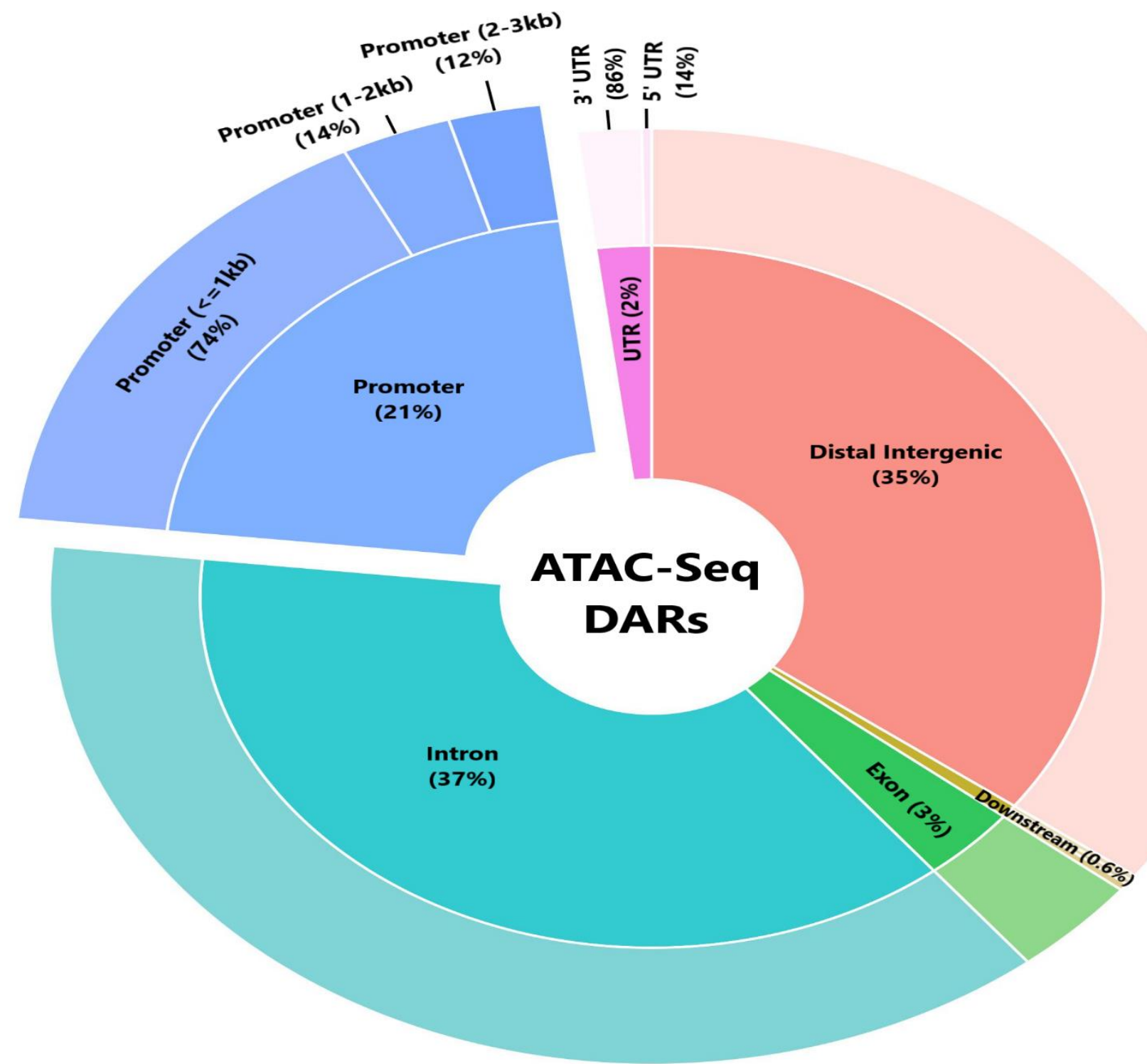

Figure 1b Annotated open significant chromatin peaks of melanocytes expressing ectopic BORIS vector. ATAC-Seq peaks of nucleosome free region (NFR) were annotated to the closest genomic region, within a window around a gene's transcriptional start site (TSS). Genomic regions distribution of Assay for Transposon-Accessible Chromatin (ATAC-seq) open chromatin peaks. All peaks obtained from a differential analysis comparing MM057 melanoma cell line expressing a DOX inducible vector, that either contained the gene BORIS (BORpos) or an empty-vector (EVpos). The pie chart depicts the proportion of 22,594 identified differential significant peaks, across the human genome (GRCh38), and the assigned genomic regions of each peak. The inner circle parentheses next to each genomic region, are the percentage (\%) of the region out of all peaks. The outer circle parentheses of Promoter and UTR regions, represent the percentage (\%) of each sub-region out of the whole parent region term. The outer circle parentheses next to Promoter regions represent the distance from TSS. $R$ programing language with Bioconductor package ChIPseeker with annotatePeak function was used for characterization of the annotation distribution of peaks to genomic regions. Significant peaks were defined as Differential Accessible Regions (DARs) by the multiple testing correction of Benjamini-Hochberg False Discovery Rate (FDR) with a cut-off 


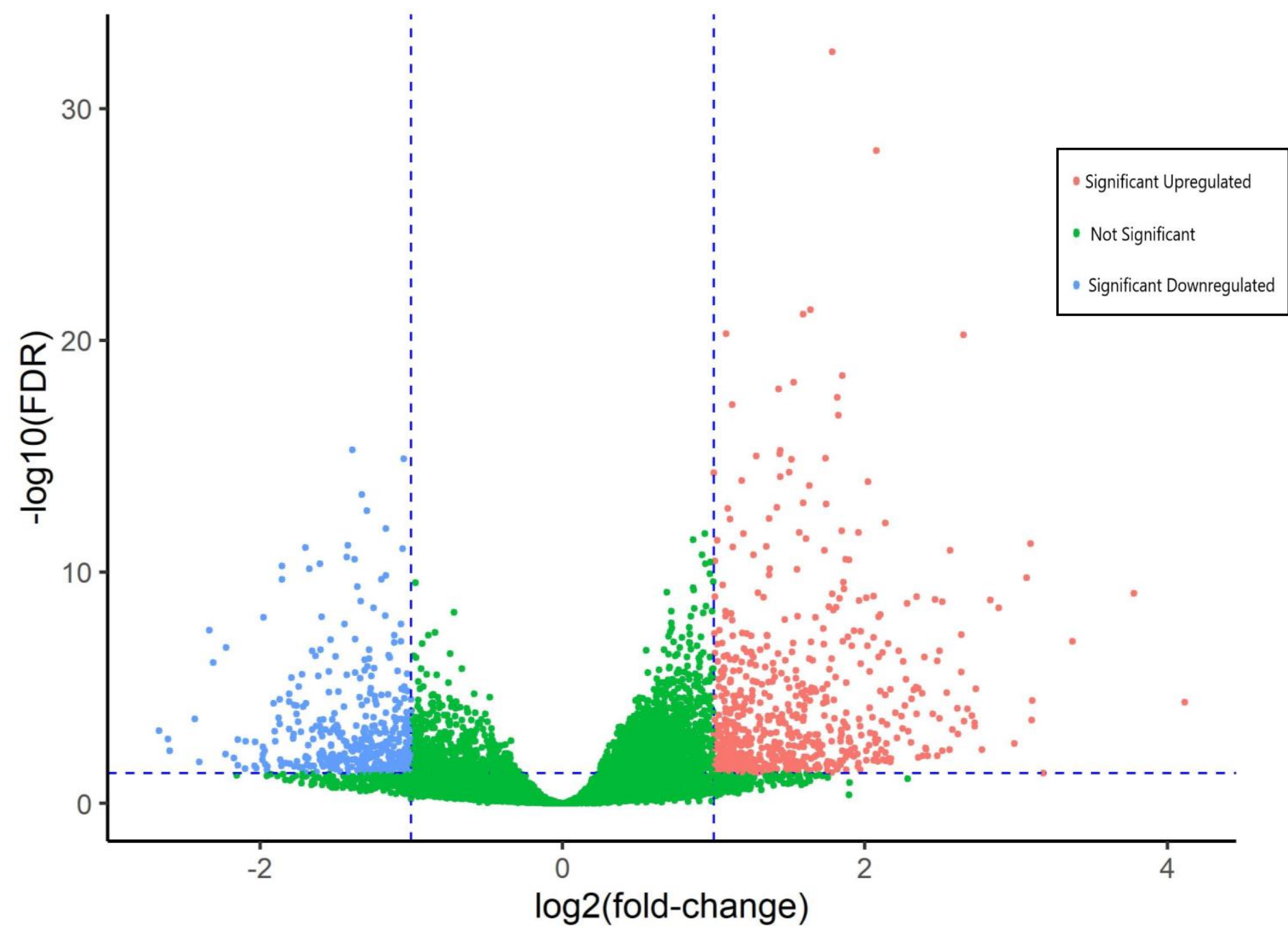

Figure 2 BORIS ectopic expression in melanoma cells leads to differentially accessible open chromatin of genes promoter's regions. Volcano plot of results ATAC-seq Differential peaks analysis between BORpos to EVpos samples. Only Peaks which annotated to promoters (0-3kb from TSS) were selected to further analysis. All promoters annotated peaks, corresponded to 16,161 genes.

Y-axis; $\log 10$ (BH-FDR) (Benjamini-Hochberg - False Discovery Rate)

$X$-axis; log2fold-change result of a differential analysis between BORpos to Evpos samples. Blue dashed lines; $X$-axis at $X=1, X=-1$. Y-axis, $Y=1.30102999566$ (FDR=0.05).

Colored in green; 12,345 not significant genes (FDR $>0.05$ and $|\log 2 \mathrm{FC}|<1)$

In red; 651 significant upregulated genes promoters (FDR $<0.05$ and $\log 2 \mathrm{FC}>1$ )

In blue; 393 significant downregulated genes promoters (FDR $<0.05$ and $\log 2 \mathrm{FC}<-1$ )

For the differential analysis Bioconductor DESeq2 was used 
phagocytosis

regulation of stem cell differentiation phagosome maturation response to oxygen-containing compound Fc-gamma receptor signaling pathway involved in phagocytosis positive regulation of signaling substrate-dependent cell migration, cell extension regulation of translation cell-substrate junction assembly cellular response to oxygen-containing compound tissue remodeling negative regulation of $T$ cell activation negative regulation of $\mathrm{T}$ cell proliferation lysosomal transport post transcriptional regulation of gene expression endocytosis epithelial cell proliferation positive regulation of GTPase activity negative regulation of ERK1 and ERK2 cascade wound healing cell differentiation
Downregulated Upregulated

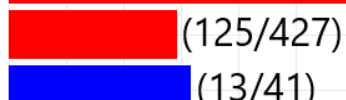

(13/41)

(126/891) (22/91)

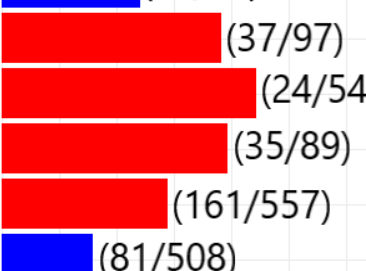

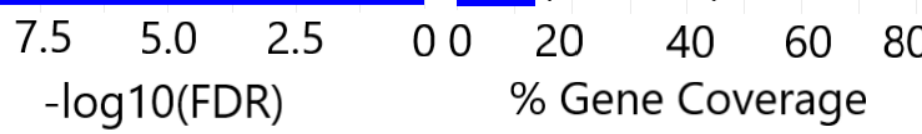

Figure 3 Functional analysis of genomic regions of significant upregulated and downregulated DARs annotated to promoters (0-3kb). Bar plots depicts selected enriched GO (gene ontology) Biological Processes (BP) terms among significant upregulated and downregulated promoters DARS (FDR $<0.05$ and $|\log 2 \mathrm{fc}|>0$ ). The promoters DARs list was consisted out of significant peaks annotated to promoters (0-3kb), upregulated DARs consisted out of 3,752 peaks, while downregulated DARs consisted out of 1,246 peaks. The rGREAT package was used for enrichment and GO_BP terms 
A

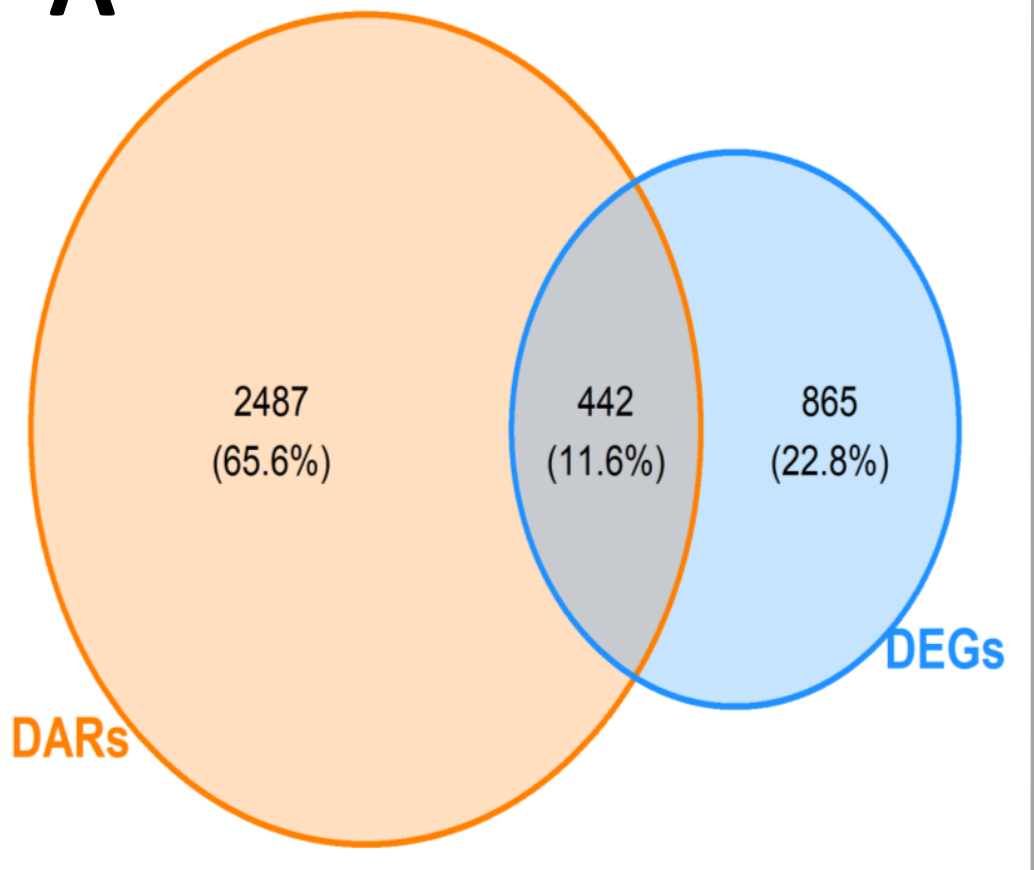

B

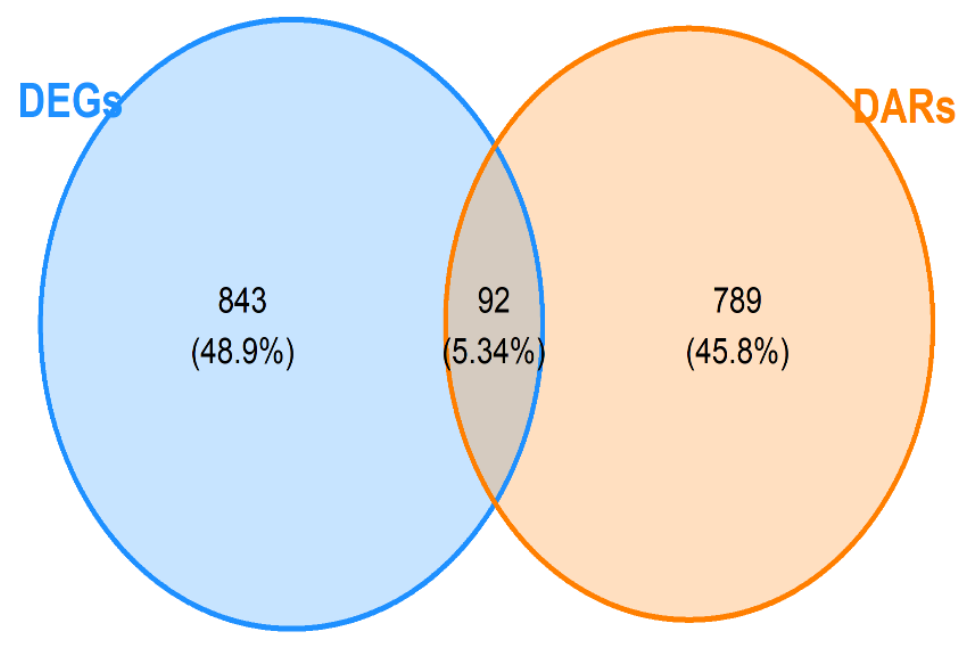

Figure 4a,b intersects results of ATAC-Seq DARS and RNA-seq DEGs resulted in $\mathbf{5 3 4}$ genes who were defined as DARs \& DEGs.

ATAC-Seq promoter (0-3kb) DARs genes and RNA-Seq Differentially expressed genes (DEGs) were intersected. Only statically significant $(F D R<0.05)$ in both genes lists were taken for the intersect analysis. This resulted in 442 upregulated genes $(\log F C>0)$ and 92 downregulated genes $(\log F C<0)$ and a total of 534 genes.

a intersect of only upregulated DARs and DEGs.

b intersects of only downregulated DARs and DEGs

both intersects had significant Chi-squared test $\left(p<1 \times 10^{-4}\right)$. 
cell migration involved in sprouting angiogenesis

regulation of cell growth

blood vessel morphogenesis

regulation of programmed cell death

positive regulation of signal transduction

positive regulation of signaling

chemotaxis

positive regulation of protein phosphorylation

regulation of cell death

cell surface receptor signaling pathway

locomotion

cell morphogenesis involved in differentiation

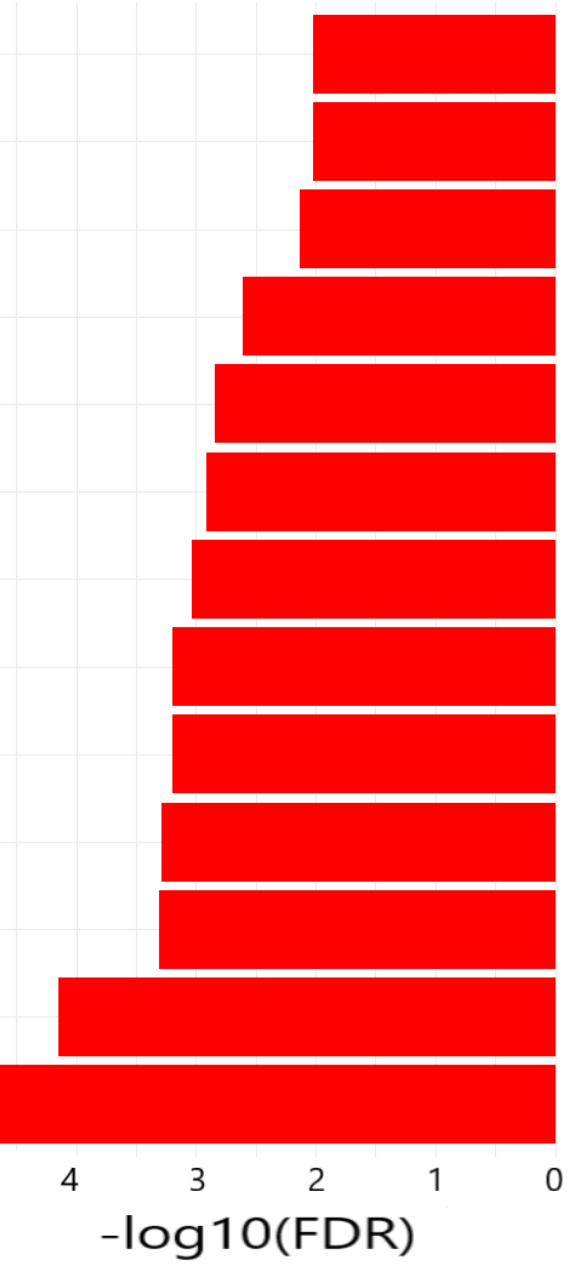

$(28 / 384)$

$(29 / 376)$

Upregulated

$(79 / 1476)$

$(85 / 1,606)$

$(37 / 496)$

$(57 / 924)$

$(86 / 1,599)$

$(108 / 2,143)$

$(70 / 1,139)$

$(41 / 491)$

10

$\%$ Gene Coverage

\section{Figure 4c Functional analysis of upregulated DARs \& DEGs genomic regions.}

Bar plots depict selected enriched GO (gene ontology) Biological Processes (BP) terms among genomic regions of genes defined as upregulated DARs and DEGs. for the enrichment analysis 554 genomic regions mapped to 442 genes with their promoter $(0-3 \mathrm{~kb})$ region identified as significant upregulated DAR, and their gene mRNA transcript was identified as upregulated DEG in the RNA-Seq experiment (i.e., genes defined as DARs \& DEGs).

The rGREAT package was used for enrichment and of the genomic regions. 
Mesenchymal cell differentiation (GO)

Epithelial Mesenchymal Transition (Hallmark)

Cell migration (GO)
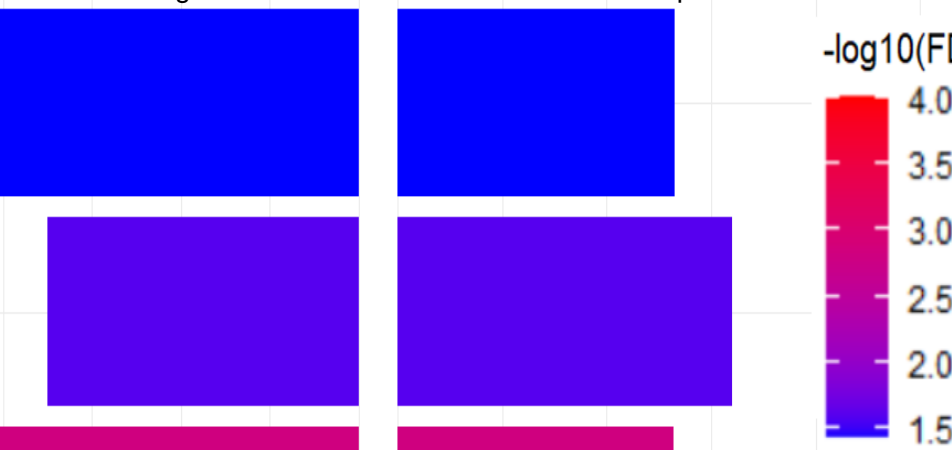

Taxis (GO)

Locomotion (GO)
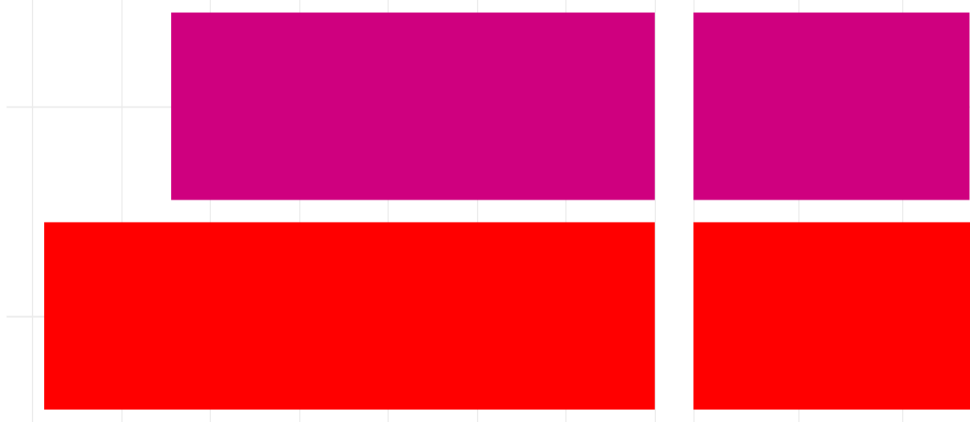

\section{Lecomotion $1 \mathrm{GO}$}

Invasive signature (Verfaillie)
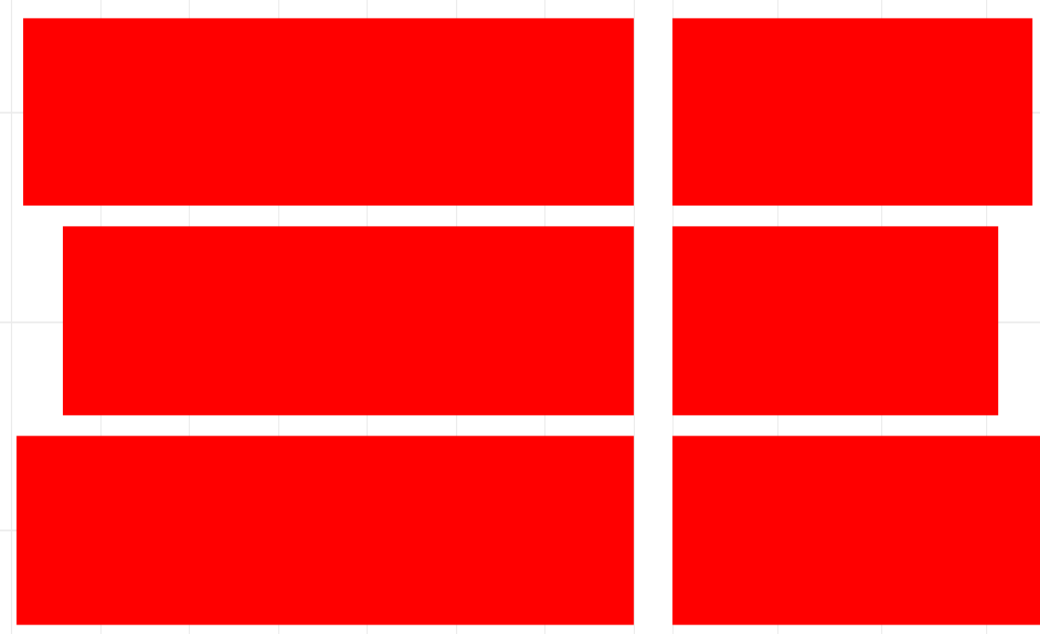

Cell cell signaling (GO)
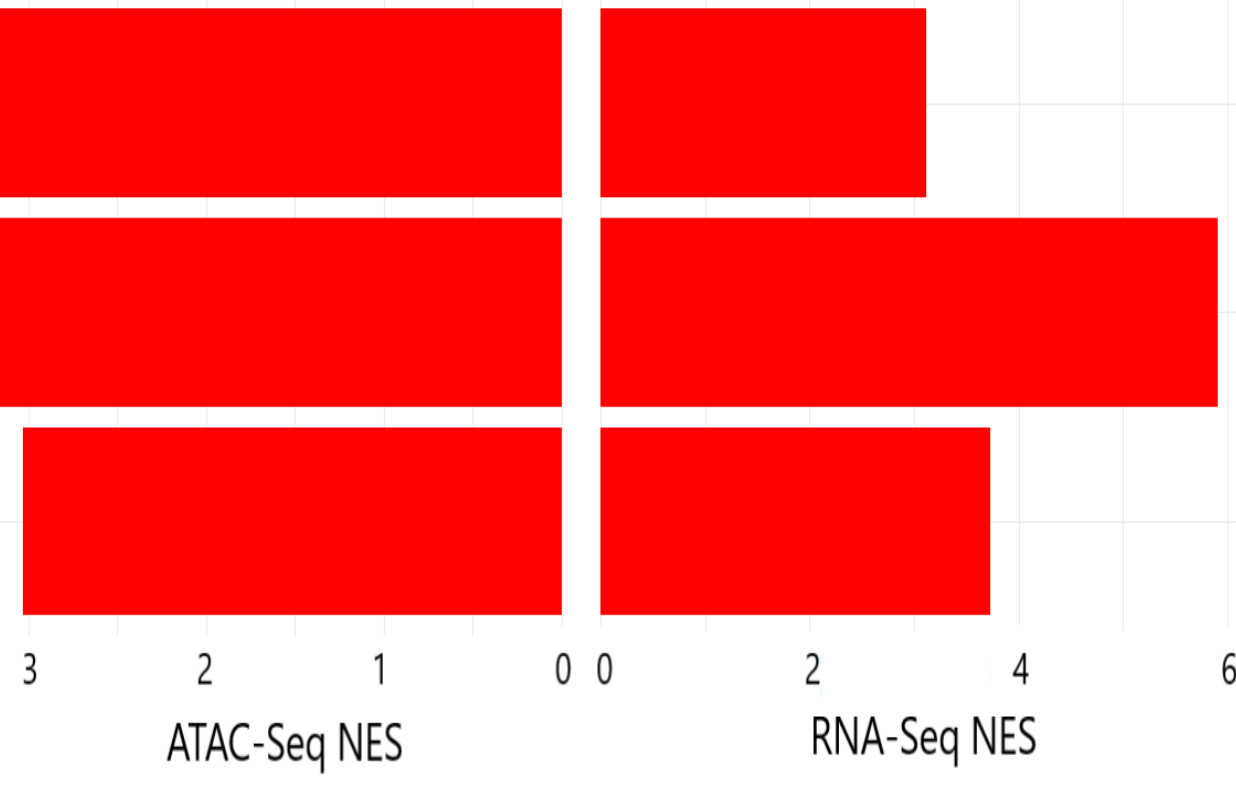

0

24

\section{Figure 5 Normalized Enrichment Score (NES) of functional analysis of ATAC-Seq peaks mapped to genes promoter (o-3kb) compared to a similar RNA-Seq based analysis.}

Bar plots depicts selected biological processes and the hallmark EMT gene set from the Molecular Signatures Database and the invasive gene signature of Verfaillie. On the left side of the plot, all 16,160 genes, which their promoter $(0-3 \mathrm{~kb})$ region had ATAC-Seq peaks who mapped to it, served as input for the Gene Set Enrichment Analysis (GSEA). On the left side of the plot, GSEA of RNA-seq data containing 16,443 genes of the same processes. In both experiments genes were ranked according to a differential analysis comparing MM057 melanoma cell line expressing a DOX inducible vector, that either contained the gene BORIS (BORpos) or an empty-vector (EVpos). The genes were ranked according to their log2FC and served as input for GSEA-pre-ranked algorithm (version 4.1.0). The bars represent Normalized Enrichment Score (NES) results of GSEA analysis. NES $>2$ is considered significant enrichment of the tested processes. The color represents -log10(FDR) as described in the legend. Peaks were annotated to their nearest gene with ChIPseeker in R. 


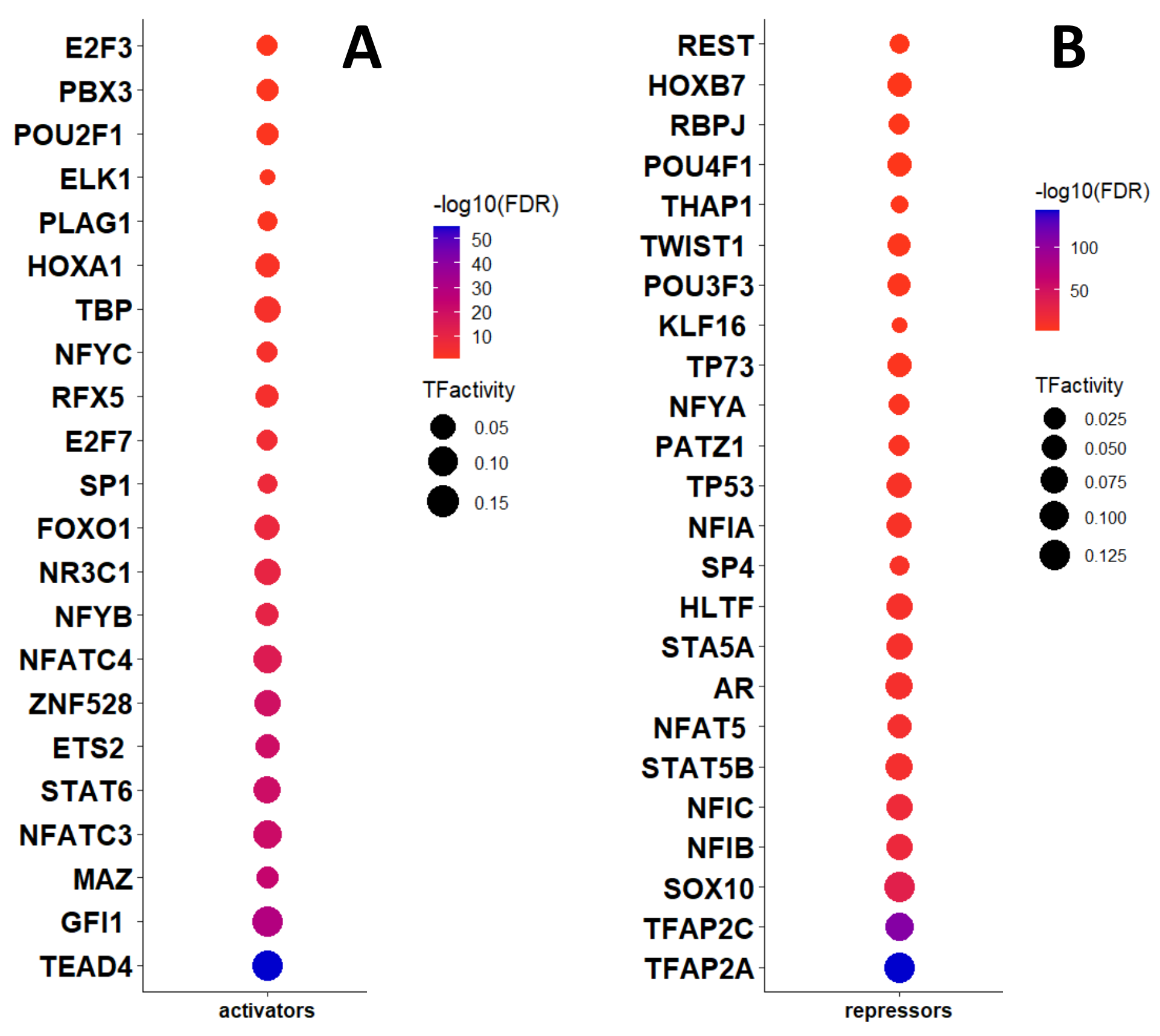

Figure 6 Results of diffTF in TF classification mode of the final selected TFs motifs that showed a significant differential activity as activators or repressors upon ectopic BORIS expression in melanoma cells.

TFs are classified as activators or repressors based on ATAC-Seq chromatin accessibility and RNA-Seq genes expression of the TFs target genes. The differential analysis was conducted between BORIS ectopic expression (BORpos) samples to the controls containing an empty vector (EVpos).

A dot plot of TFs classified as activators.

B dot plot of TFs classified as repressors.

The dot color represents - $\log 10(\mathrm{FDR})$, the dot size represents the $\triangle \mathrm{TF}$ activity as measured between BORpos to EVpos samples. The TFs are ordered by statical significant were the most significant are at the bottom of the plot. 


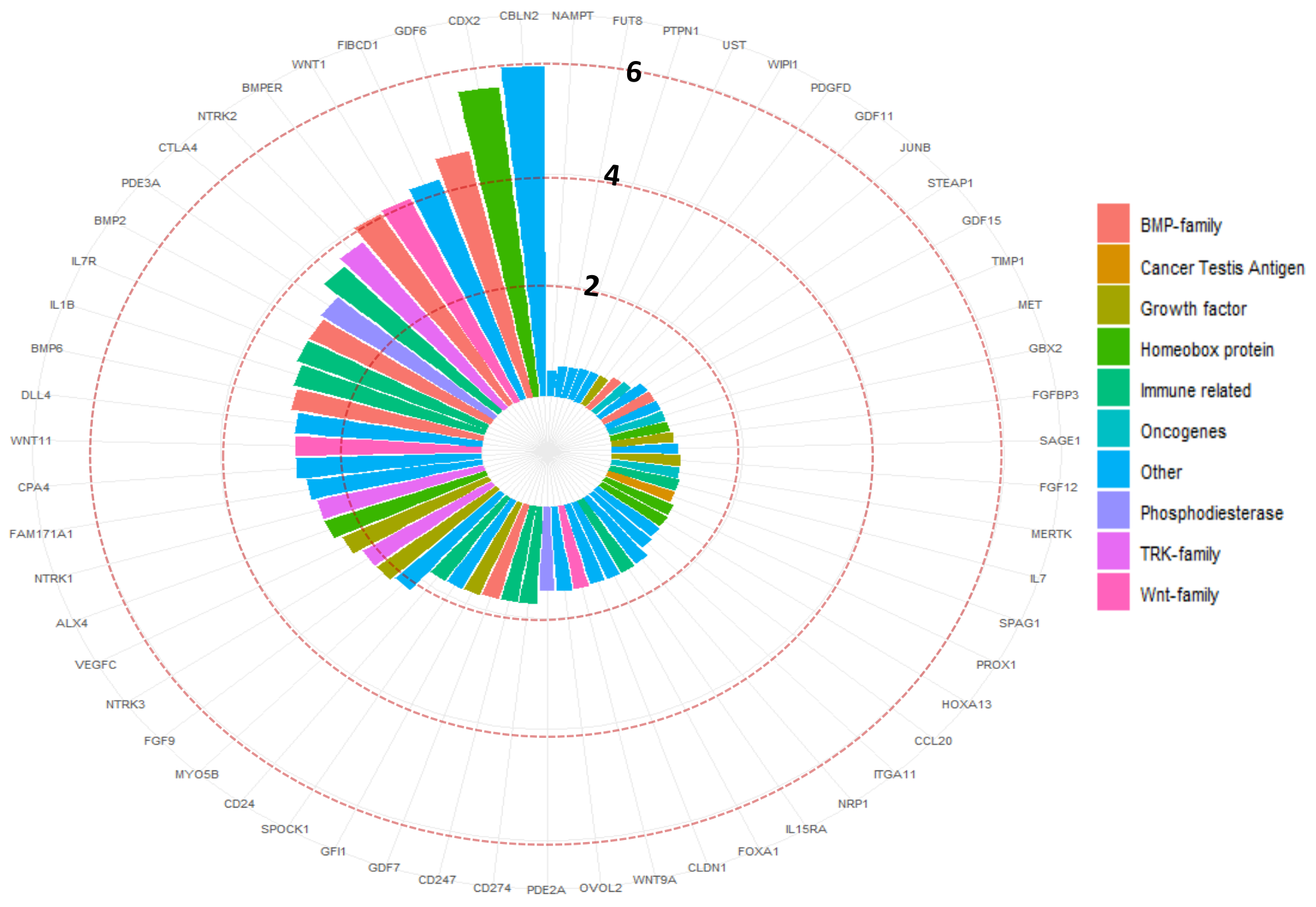

Figure 7 Circular bar Plot of 59 significant tumor progressors genes, who were identified as DARs \& DEGs. The genes are grouped by biological family or function if available.

Other group represents genes which do not belong to any particular pathway or attributed to a protein family

$X$-axis (outer circle characters); each gene's name corresponding to its bar. $\mathrm{Y}$-axis (inner circle bars); each bar represents the average log2FC (fold change) results of differential analyses of ATAC-seq DARs and RNA-Seq DEGs between BORpos samples to EVpos. Light red dash line at $\log 2 \mathrm{FC}=2,4,6$. 


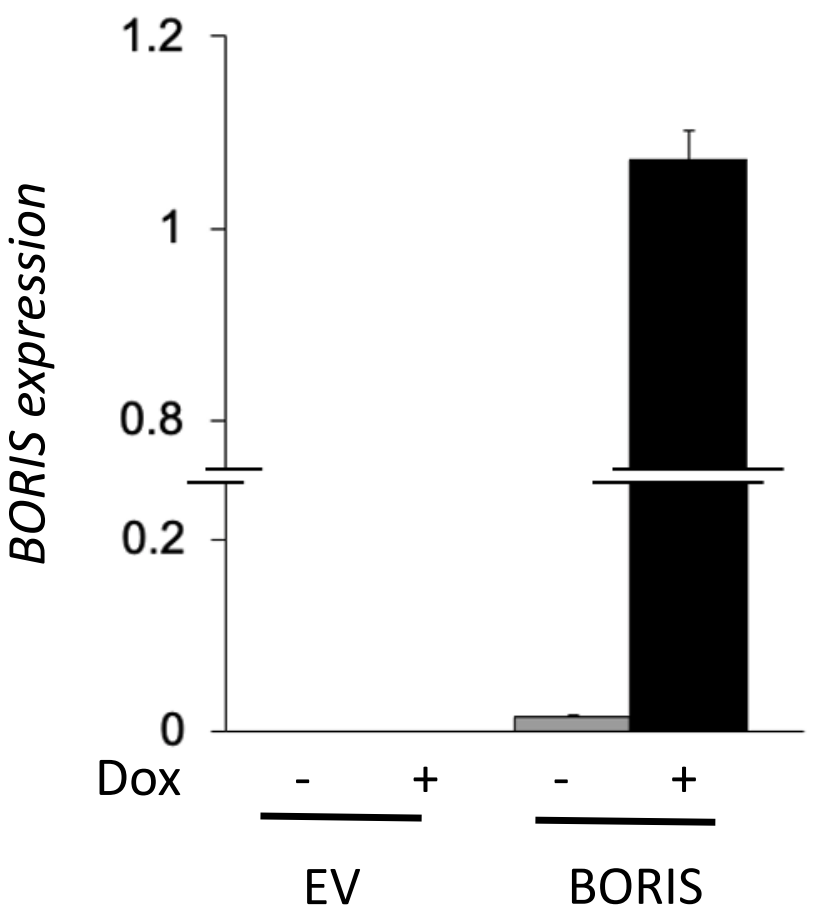

B

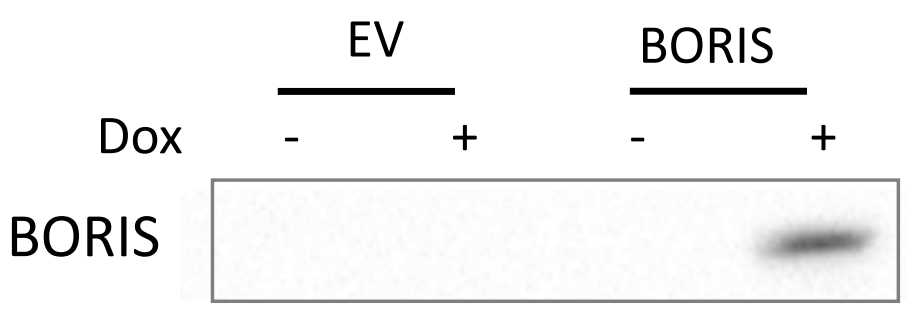

Tubulin

Supplement 1 Validation of ectopic BORIS expression in melanoma cells

$(\mathrm{a}, \mathrm{b}) \mathrm{BORIS}$ expression was induced in the MM057 cell line using $50 \mathrm{ng} / \mathrm{ml}$ dox for 5 days. a) Expression of BORIS mRNA as determined by qPCR. A technical triplicate was performed and expression was normalized to HPRT1 and TBP. Error bars represent the standard error of the mean. b) Expression of BORIS protein as determined by immunoblot. Anti-Tubulin was used as a loading control. Dox doxycycline, 

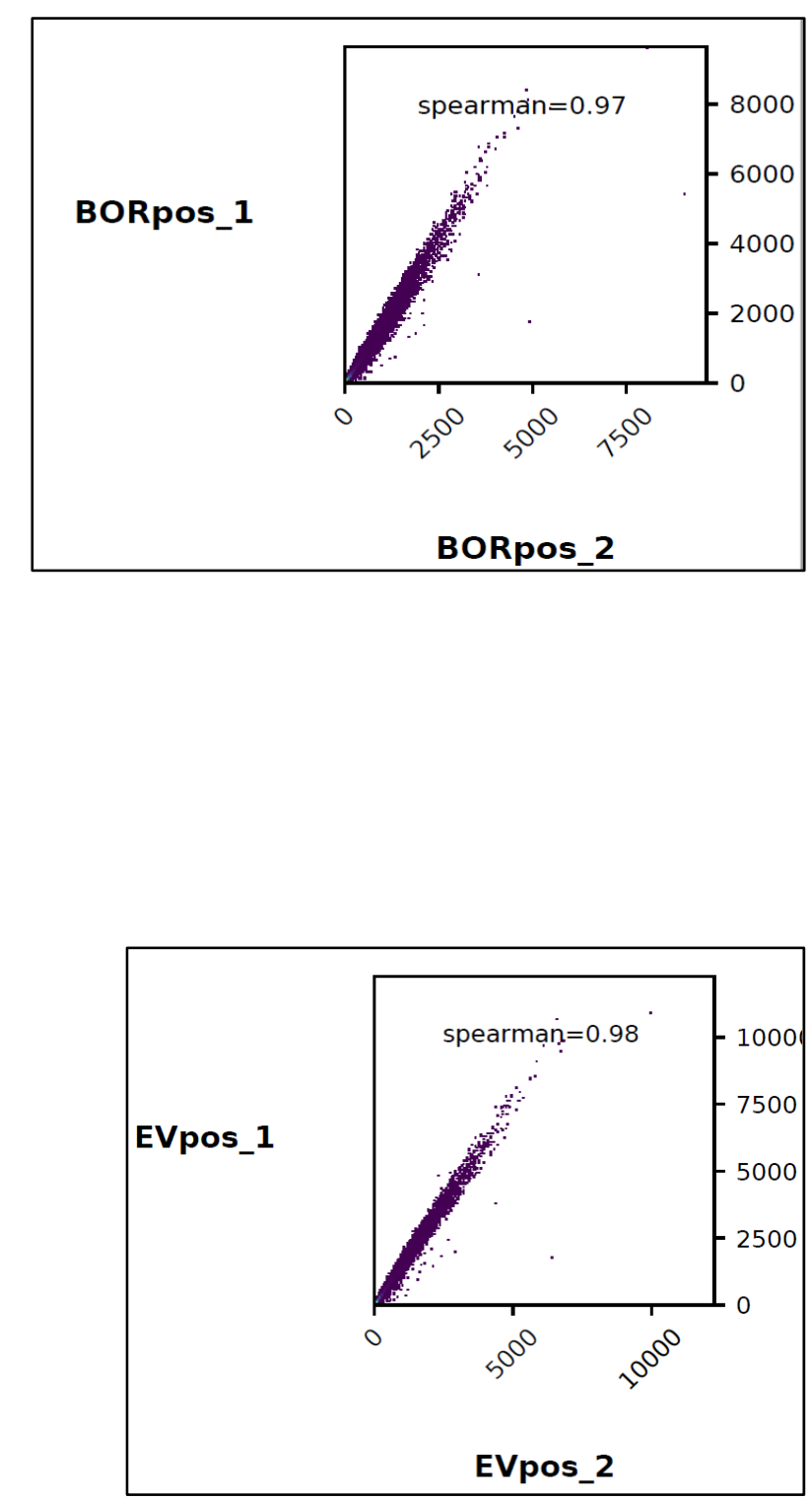
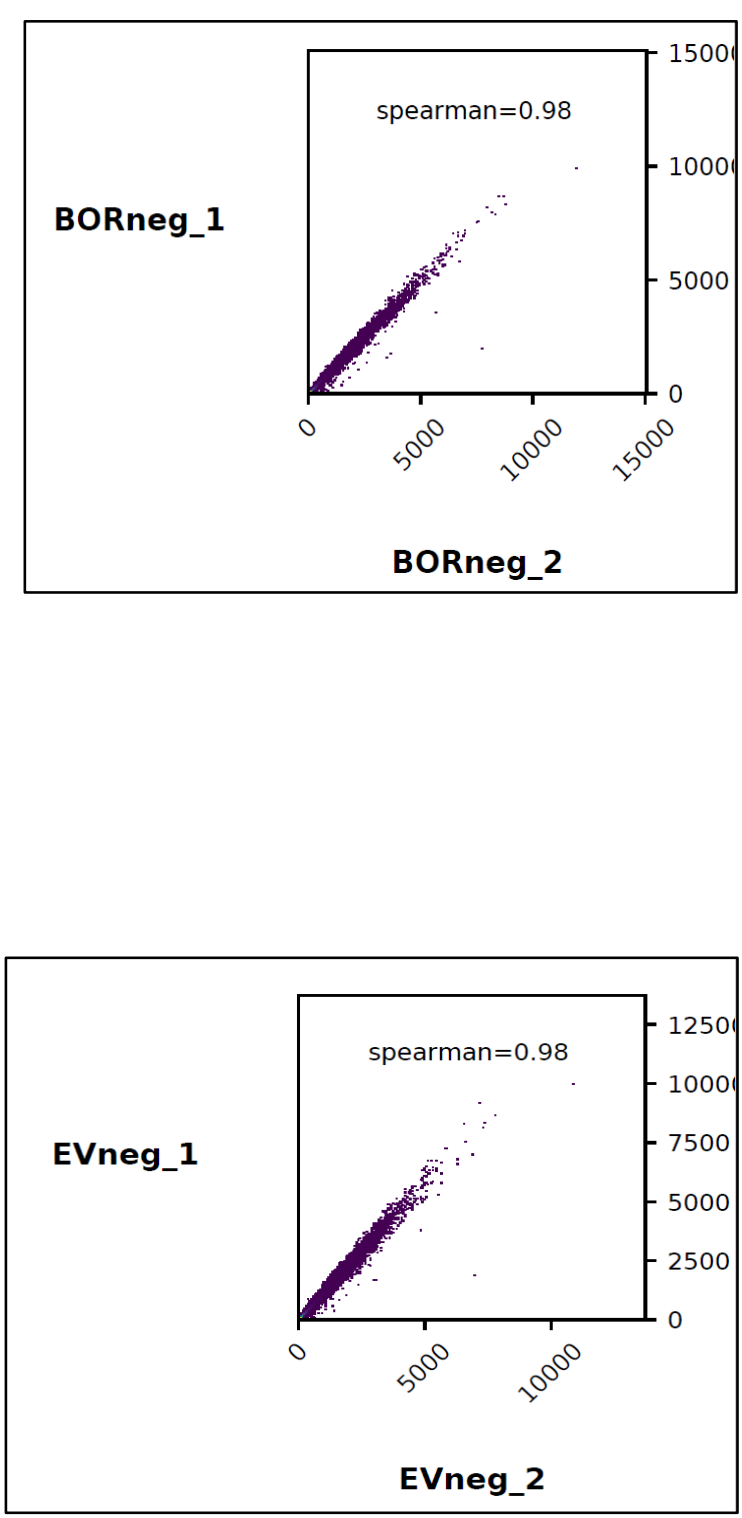

Supplement 2 Correlation between same condition replicates based on ATAC-Seq initial

BAM files (before sifting the reads)

$B O R=B O R I S, E V=E m p t y$ Vector, NEG= without

Doxycycline,$P O S=$ with Doxycycline

1=biological repeat number 1

$2=$ biological repeat number 2 


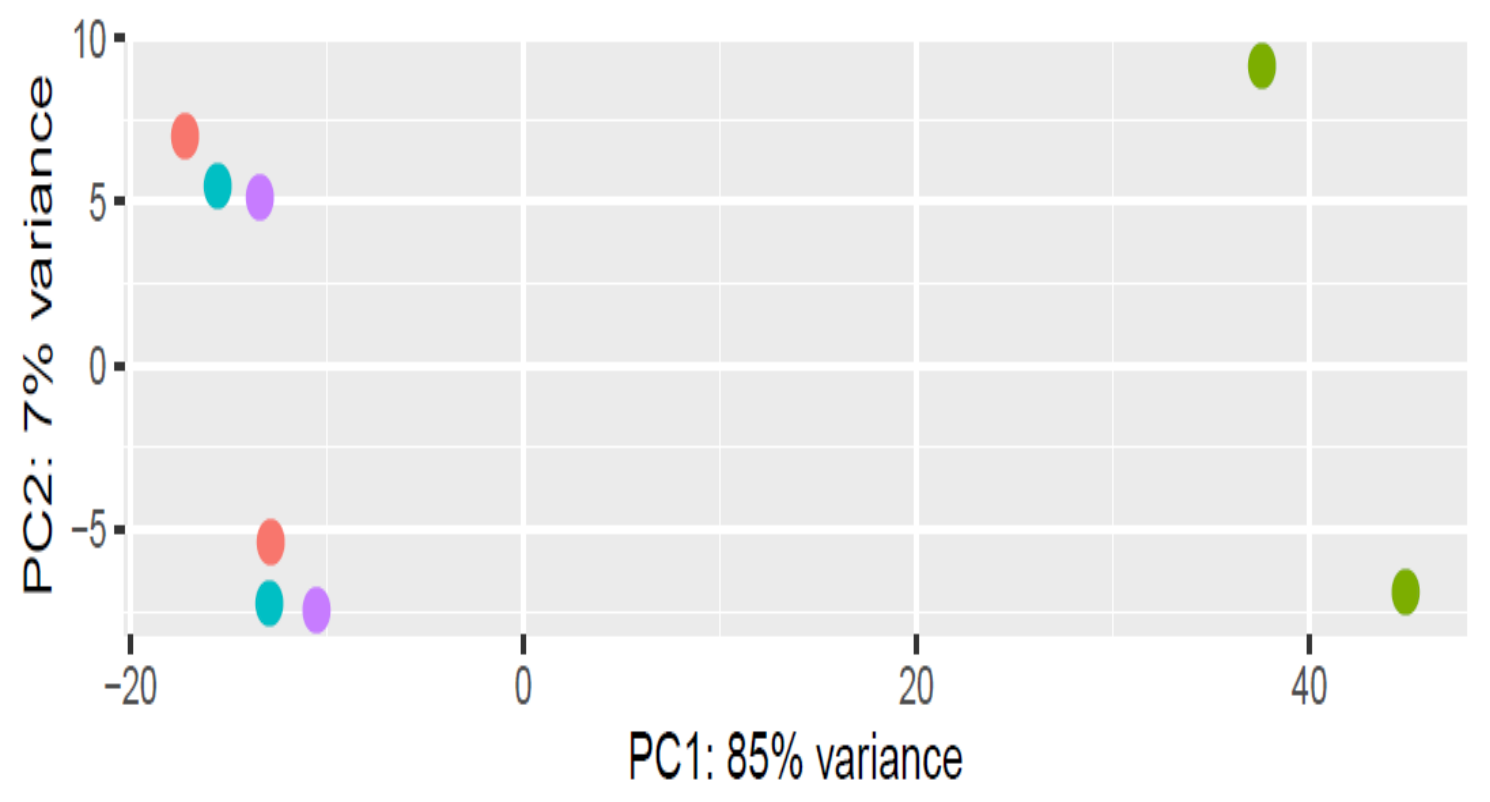

Supplement 3 Principal Component Analysis (PCA) of the four conditions of the ATAC-seq samples.

$\mathrm{BOR}=\mathrm{BORIS}, \mathrm{EV}=\mathrm{Empty}$ Vector, NEG= without Doxycycline, $\mathrm{POS}=$ with Doxycycline 


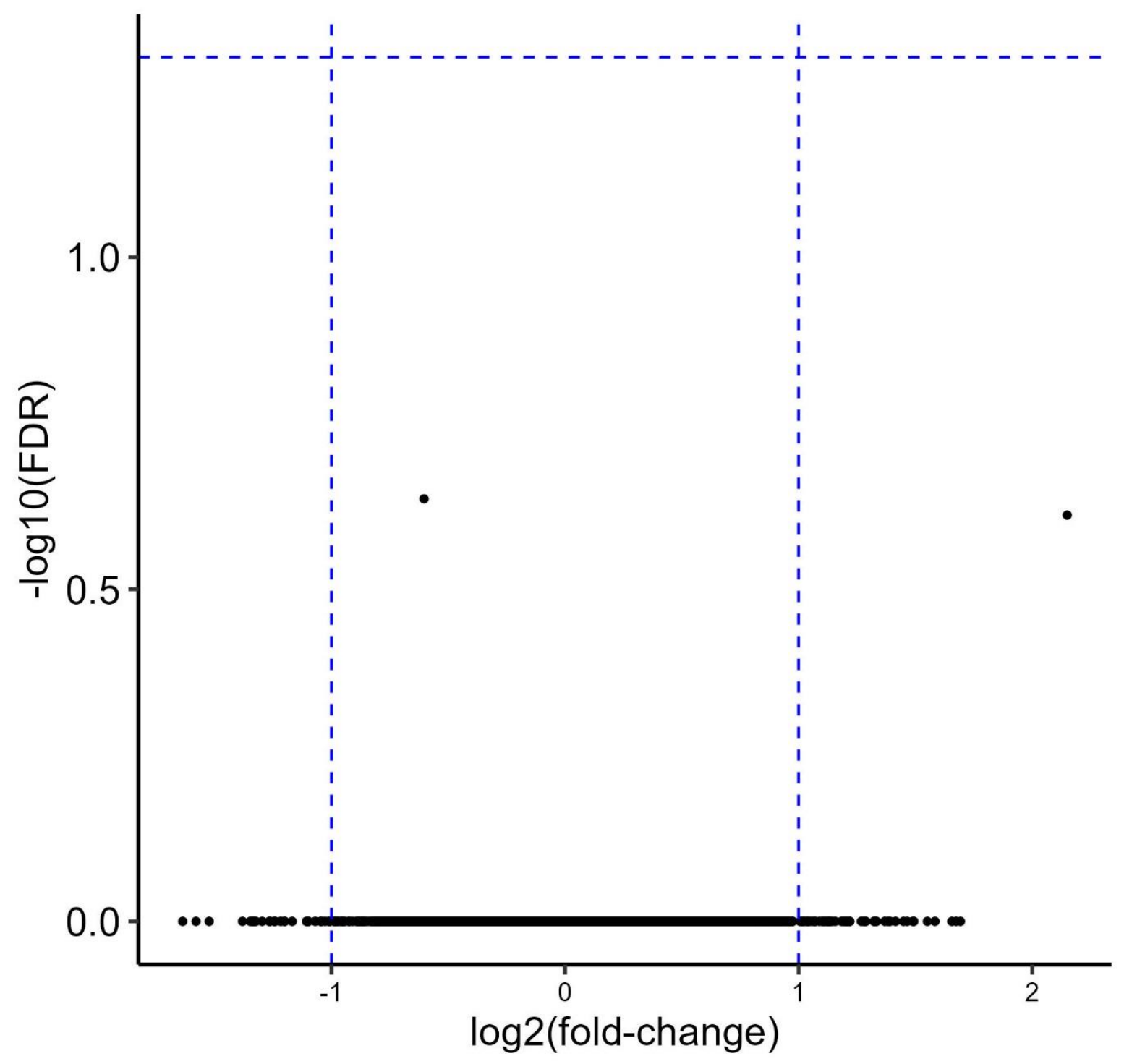

Supplement 4 Empty-Vector (EV) expression in melanoma cells leads to no significant differentially accessible open chromatin.

Volcano plot of results ATAC-seq Differential peaks analysis between EVRpos to EVneg samples. Differentially accessible regions (DARs) corresponded to 18,897 genes presented in the plot. Non were statistically significant $(F D R<0.05)$ up or down regulated in the EVpos samples compared to the EVneg samples. axis; $\log 10$ (BH-FDR) (Benjamini-Hochberg - False Discovery Rate).

$X$-axis; log2fold-change result of a differential analysis between EVpos to Evneg samples.

Blue dashed lines; $X$-axis at $X=1, X=-1$. Y-axis, $Y=1.30102999566$ (FDR=0.05).

For the differential analysis Bioconductor DESeq2 was used. 


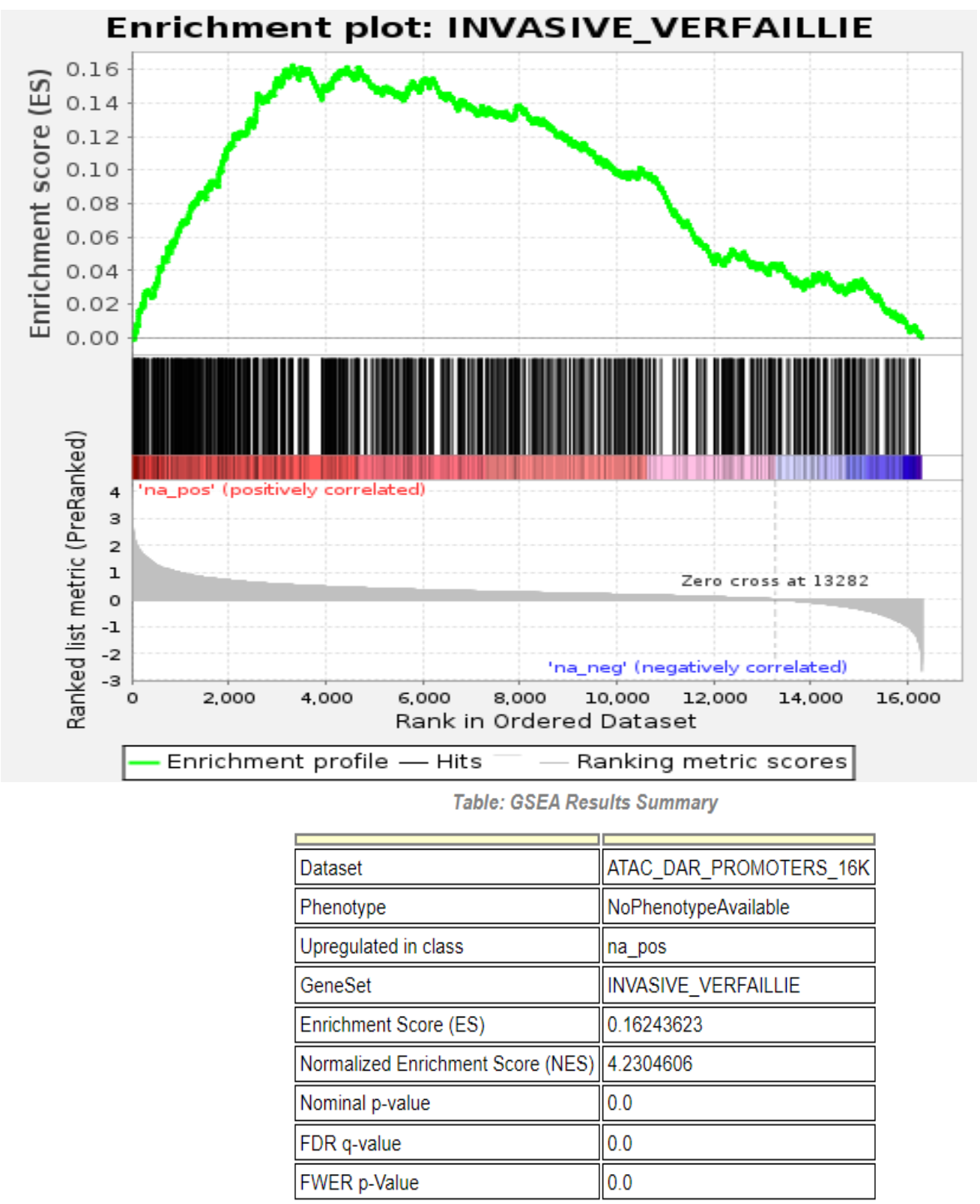

Supplement 5 Verfaille invasive gene set signature barcode plots of all ATAC-Seq peaks identified.

All 16,161 genes, which their promoter (0-3kb) region had ATAC-Seq peaks who mapped to it, served as input for the Gene Set Enrichment Analysis (GSEA). Genes were ranked according to a differential analysis comparing MM057 melanoma cell line expressing a DOX inducible vector, that either contained the gene BORIS (BORpos) or an empty-vector (EVpos). The genes were ranked according to their log2FC and served as input for GSEA-pre-ranked algorithm (version 4.1.0). The bars represent Normalized Enrichment Score (NES) results of GSEA analysis 


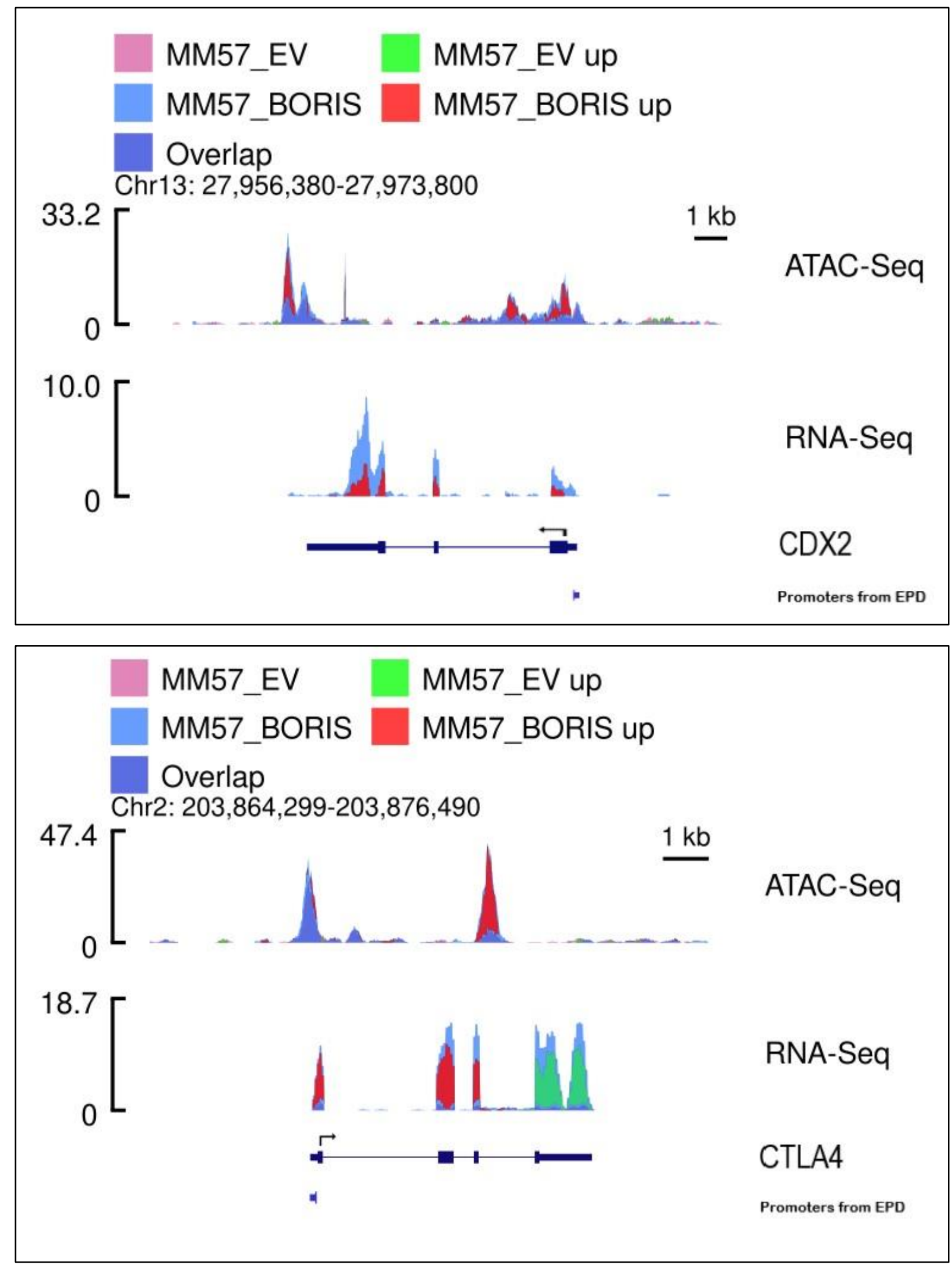



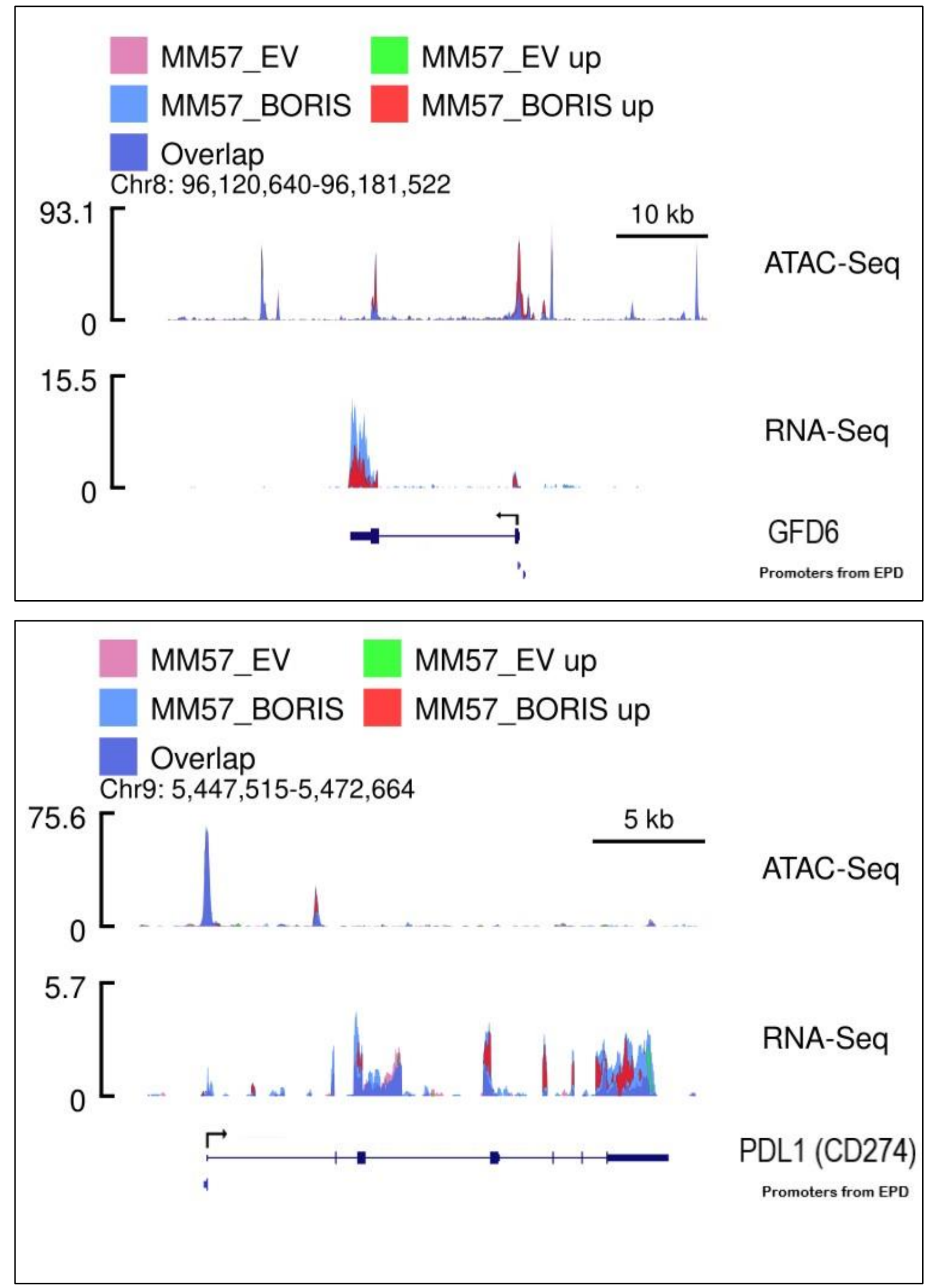


\section{Supplement 6 TAC-Seq and RNA-Seq genomic Tracks of the main genes identified as DARs \& DEGs.}

CDX2,CTLA4,GDF6,PDL-1 genomic regions Tracks of ATAC-seq and RNA-Seq data were made using Spark, a python tool NGS Visualization Tool. The input of the tool was bedgraph files generated from ATAC-Seq and RNA-Seq BAM files. The tracks represent the average of the repats of each condition. Regions in the genome where BORpos samples had significantly more reads are marked in red. Those areas in the genome, which samples are defined as areas where the delta of the means is greater than the sum of standard deviations of controls (EVpos) and treatment (BORpos) files. 


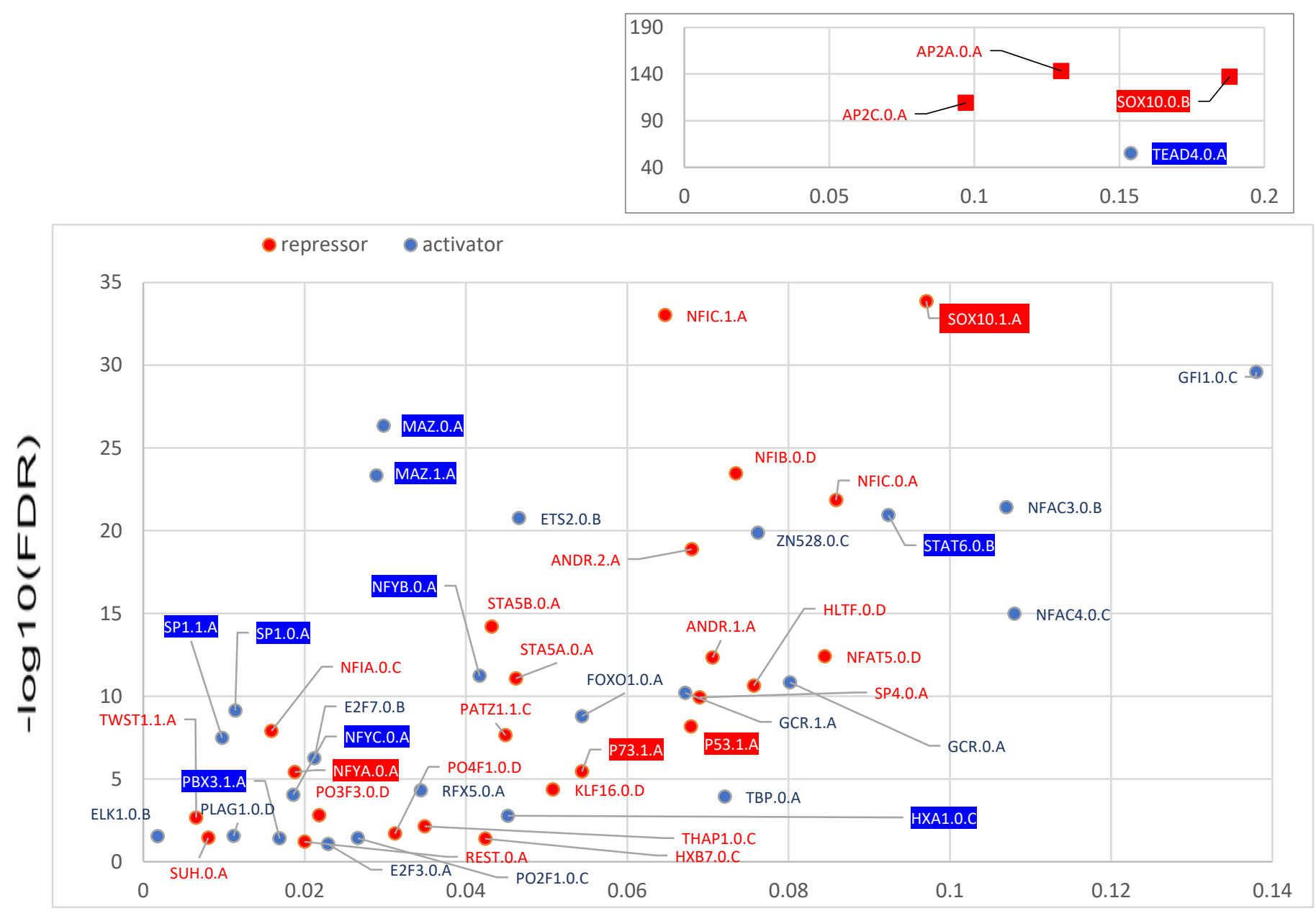

$\Delta T F$ activity (BORIS vs Empty-Vector)

Supplement 7 DiffTF motifs of significant transcription factors differentially activated/repressed upon ectopic BORIS expression in melanoma cells In red are repressors TFs motifs, and in blue activators TFs motifs. Highlighted, are known cancer related TFs motifs $X$ axis; $\triangle T F$ activity tested in BORpos vs Evpos. Y-axis; -log10(adj. p-value).

TFs motif were taken from https://hocomoco11.autosome.ru/ 
bioRxiv preprint doi: https://doi.org/10.1101/2022.02.11.479460; this version posted February 11, 2022. The copyright holder for this preprint (which was not certified by peer review) is the author/funder. All rights reserved. No reuse allowed without permission.
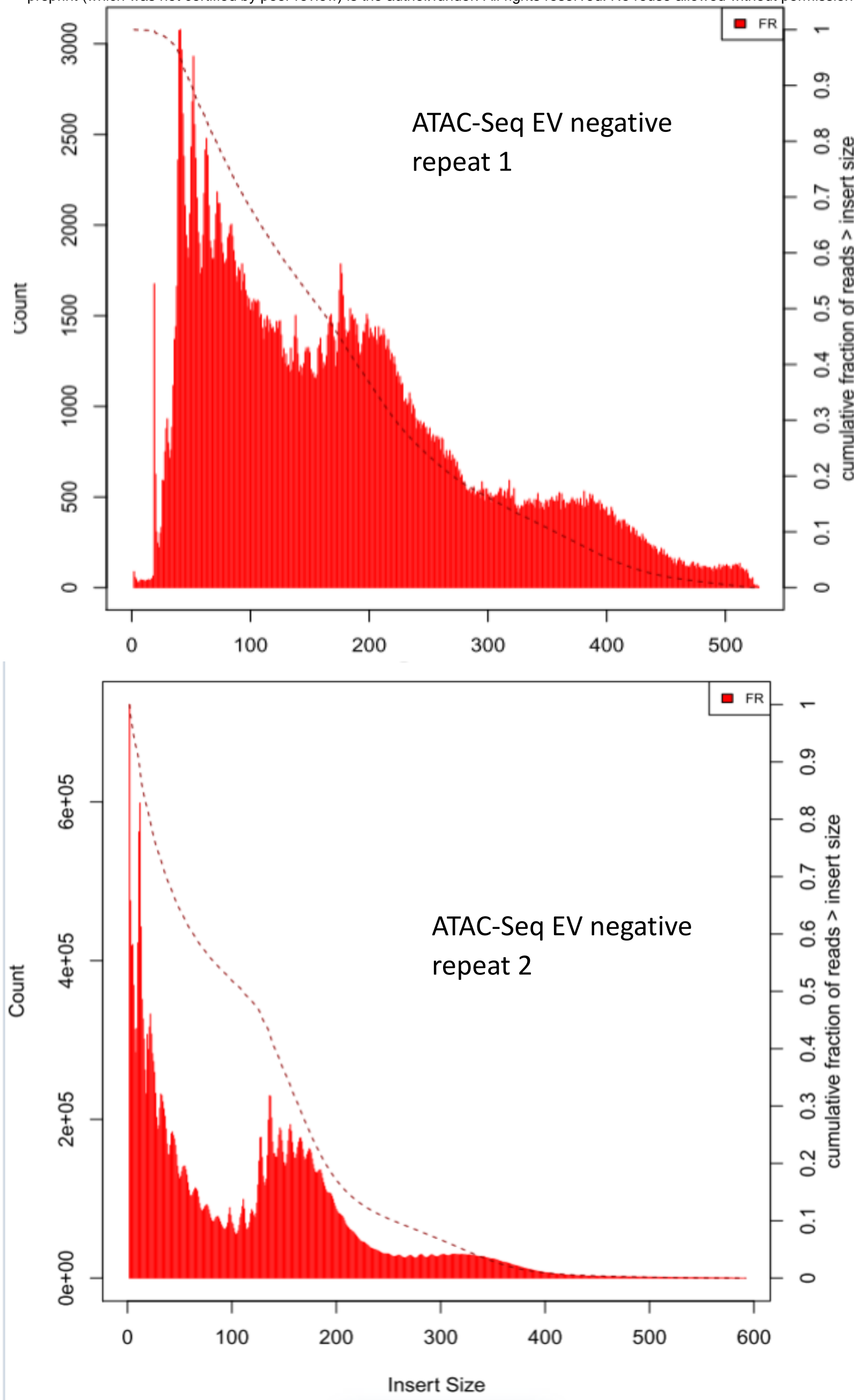

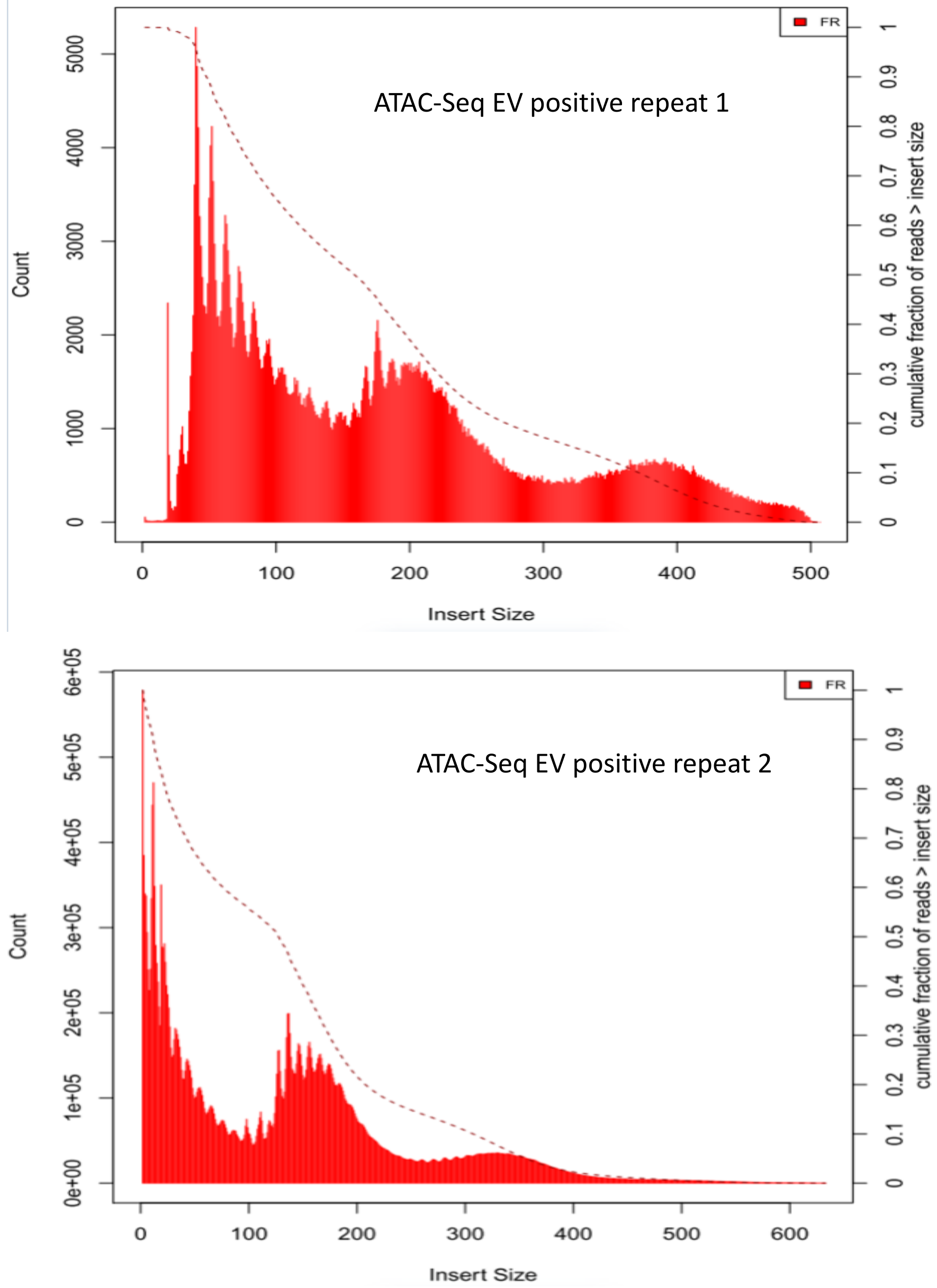

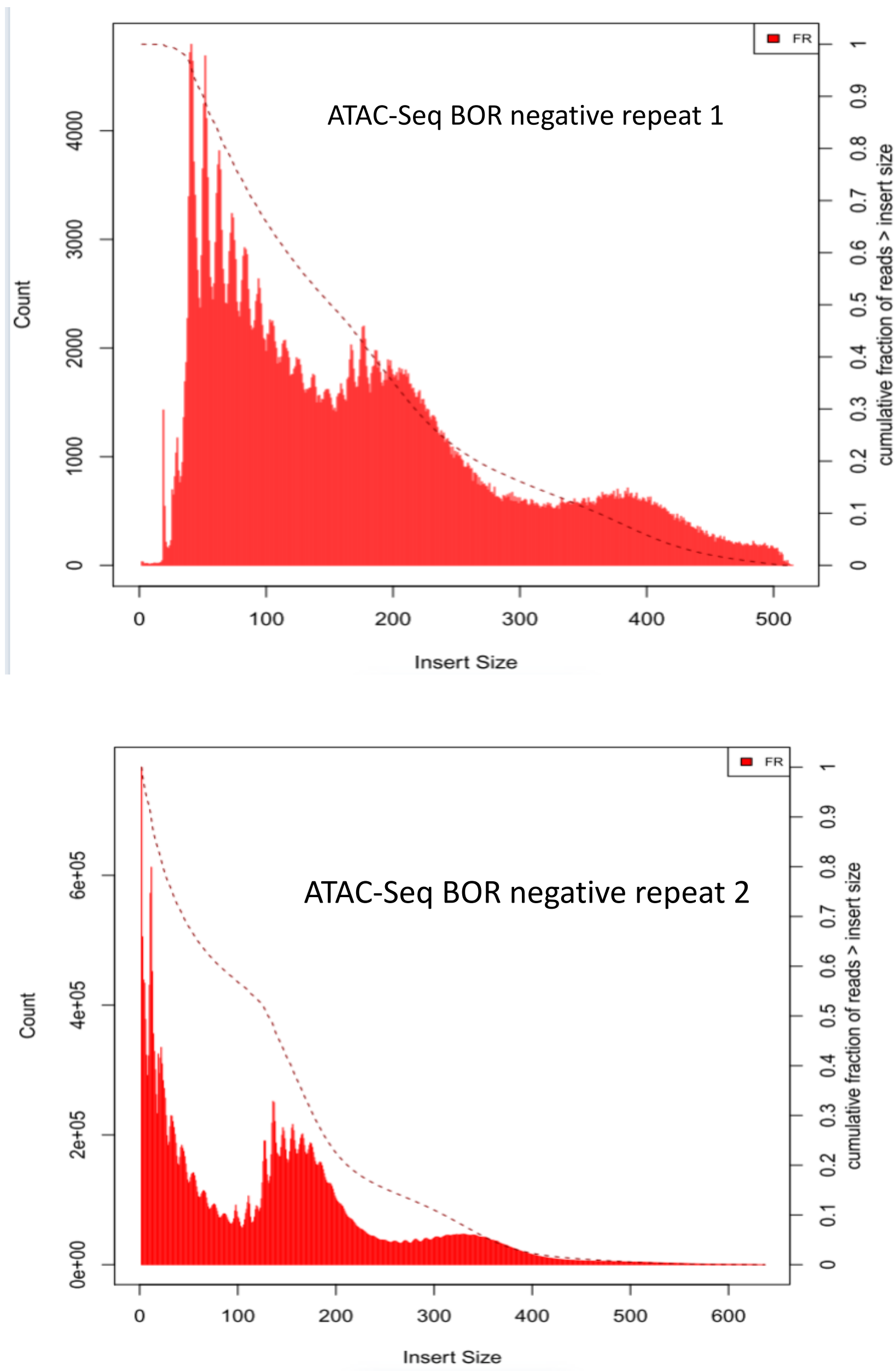

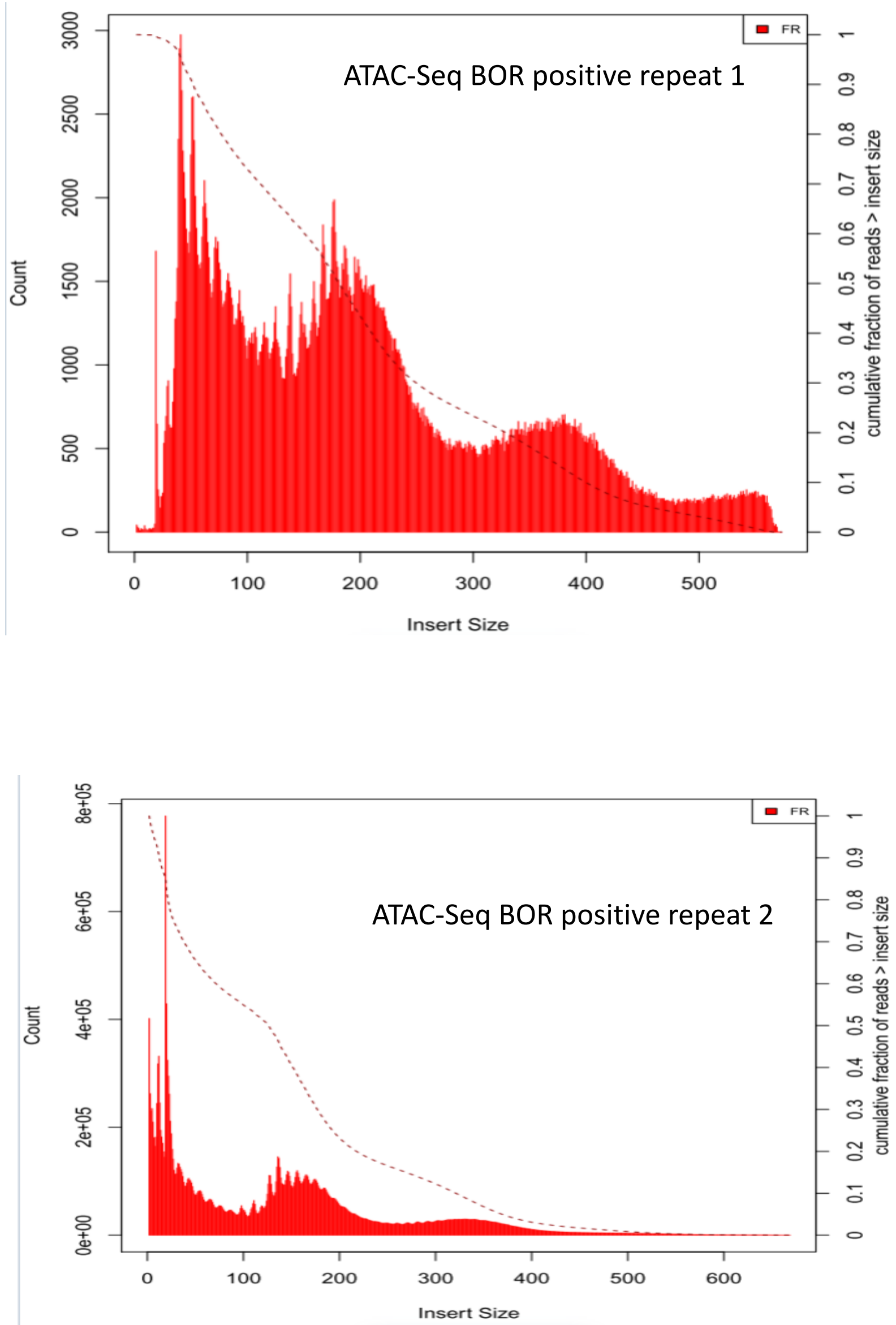
Supplement 8 Distribution of ATAC-Seq fragment size counts, over the conditions repeats. As given by PicardTools: CollectInsertSizeMetrics.

The $\mathrm{X}$-axis represents the number of fragments.

The $\mathrm{Y}$-axis represent the fragments size in base-pairs (bp). 
A)

Library \#1

Library \#2

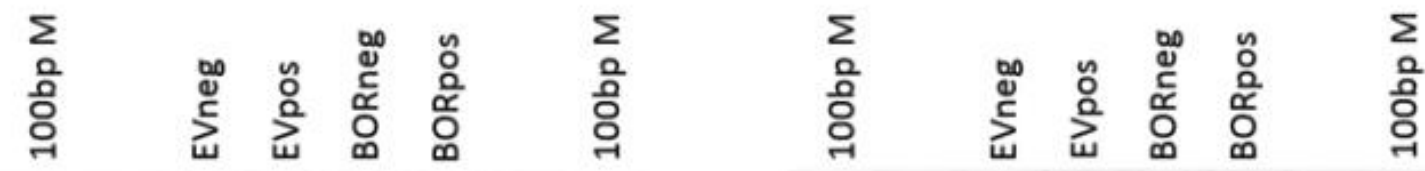

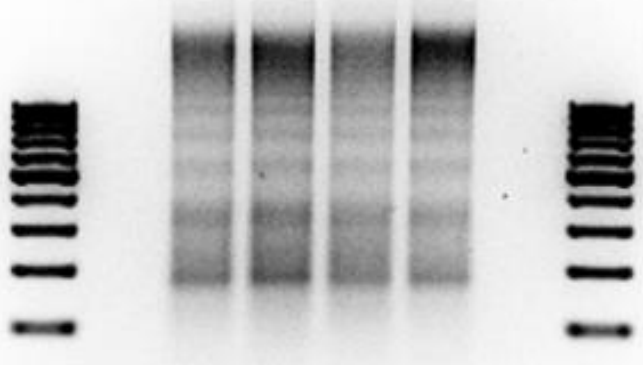

B)

\begin{tabular}{|l|cccc|}
\hline & EVneg & EVpos & BORneg & BORpos \\
\hline GAPDH & 21.345 & 21.495 & 22.125 & 21.915 \\
KAT6B & 21.275 & 21.02 & 21.27 & 21.115 \\
SLC22A3 & 27.075 & 27.06 & 27.61 & 27.175 \\
RHO & 25.765 & 25.655 & 26.125 & 26.185 \\
\hline
\end{tabular}

\begin{tabular}{|l|cccc|}
\hline & EVneg & EVpos & BORneg & BORpos \\
\hline GAPDH & 21.285 & 20.485 & 20.65 & 20.255 \\
KAT6B & 20.425 & 19.795 & 20.075 & 20.025 \\
SLC22A3 & 26.945 & 25.815 & 26.05 & 25.785 \\
RHO & 25.39 & 24.425 & 24.73 & 24.825 \\
\hline
\end{tabular}

C)

\begin{tabular}{|l|cccc|}
\hline mtDNA & EVneg & EVpos & BORneg & BORpos \\
\hline WCL & 8.83 & 9.38 & 10.24 & 9.64 \\
Library \#1 & 14.16 & 14.34 & 14.14 & 14.56 \\
Library \#2 & 13.03 & 12.51 & 12.46 & 13.54 \\
\hline
\end{tabular}

\begin{tabular}{|l|cccc|}
\hline ND1-mtDNA & EVneg & EVpos & BORneg & BORpos \\
\hline WCL & 8.45 & 8.88 & 9.49 & 9.27 \\
Library \#1 & 13.68 & 13.89 & 13.55 & 13.92 \\
Library\#2 & 12.06 & 11.99 & 11.92 & 12.99 \\
\hline
\end{tabular}

\section{Supplement 9 ATAC-seq library quality control.}

A) Part of the DNA from each ATAC-seq library was analyzed by agarose gel to verify the presence of a nucleosome pattern. $B$ and $C$ ) Quantitative cycle (Cq) value for each target as assessed by qPCR on DNA from ATAC-seq libraries. B) Primers for GAPDH and KAT6B were used to amplify regions of open chromatin and SLC22A3 and RHO for regions of closed chromatin. C) Two sets of primers (mtDNA and ND1$m t D N A$ ) were used to amplify mtDNA. WCL: library prepared from whole cell lysate instead of isolated nuclei. 


\begin{tabular}{|c|c|}
\hline Primer name & Sequence (5'-3') \\
\hline \multicolumn{2}{|c|}{ qPCR primers } \\
\hline HPRT1_267F & AGGGTGTTTATTCCTCATGGAC \\
\hline HPRT1_367R & CACAGAGGGCTACAATGTGAT \\
\hline BORIS-1590F & CGCTCACTTCAGGAAATACCAC \\
\hline BORIS-1696R & CCGAATGTCTGTGCAGGTTAAT \\
\hline TBP_562F & GAAACGCCGAATATAATCCCAA \\
\hline TBP_672R & GGCTCCTGTGCACACCATT \\
\hline \multicolumn{2}{|c|}{ ATAC-seq Nextera primers } \\
\hline Ad1_noMX & $\begin{array}{l}\text { AAT GAT ACG GCG ACC ACC GAG ATC TAC ACT } \\
\text { CGT CGG CAG CGT CAG ATG TG }\end{array}$ \\
\hline Ad2.3_AGGCAGAA & $\begin{array}{l}\text { CAA GCA GAA GAC GGC ATA CGA GAT TTC TGC } \\
\text { CTG TCT CGT GGG CTC GGA GAT GT }\end{array}$ \\
\hline Ad2.4_TCCTGAGC & $\begin{array}{l}\text { CAA GCA GAA GAC GGC ATA CGA GAT GCT CAG } \\
\text { GAG TCT CGT GGG CTC GGA GAT GT }\end{array}$ \\
\hline Ad2.5_GGACTCCT & $\begin{array}{l}\text { CAA GCA GAA GAC GGC ATA CGA GAT AGG } \\
\text { AGT CCG TCT CGT GGG CTC GGA GAT GT }\end{array}$ \\
\hline Ad2.6_TAGGCATG & $\begin{array}{l}\text { CAA GCA GAA GAC GGC ATA CGA GAT CAT } \\
\text { GCC TAG TCT CGT GGG CTC GGA GAT GT }\end{array}$ \\
\hline \multicolumn{2}{|c|}{ ATAC-seq control primers } \\
\hline KAT6B_213F_ATAC & CAGCGGTCTGTTGATTGGTAT \\
\hline KAT6B_381R_ATAC & GCCTACGGGTCTGTCTGTCA \\
\hline GAPDH_130F_ATAC & ACGTAGCTCAGGCCTCAAGA \\
\hline GAPDH_237R_ATAC & GGCTCAATTTATAGAAACCGGG \\
\hline ATACNC_RHO-F & TGGGTGGTGTCATCTGGTAA \\
\hline ATACNC_RHO-R & GGA TGGAA TGGA TCAGA TGG \\
\hline ATACNC_SLC22A3-F & GGAGAGGGTGGACAGA TTGA \\
\hline ATACNC_SLC22A3-R & TCAGCCTTGCTGCTACAGTG \\
\hline \multicolumn{2}{|c|}{ ATAC-seq mtDNA primers } \\
\hline ND1-mtDNA_F & CCT AAA ACC CGC CAC ATC T \\
\hline ND1-mtDNA_R & GAG CGA TGG TGA GAG CTA AGG \\
\hline mtDNA-ATAC_F & CTT CTG GCC ACA GCA CTT AAA C \\
\hline mtDNA-ATAC_R & AAT CTG GTT AGG CTG GTG TTA GG \\
\hline
\end{tabular}

\section{Supplement 10 Primers table}

